\title{
FORMAL SOLUTIONS AND A RELATED EQUATION FOR A CLASS OF FOURTH ORDER DIFFERENTIAL EQUATIONS OF A HYDRODYNAMIC TYPE(1)
}

\author{
BY \\ RUDOLPH E. LANGER
}

1. Introduction. This paper has as its objectives the determination of complete sets of formal solutions of a differential equation of the form

$$
\frac{d^{4} w}{d z^{4}}+\lambda^{2}\left\{P(z, \lambda) \frac{d^{2} w}{d z^{2}}+Q(z, \lambda) \frac{d w}{d z}+R(z, \lambda) w\right\}=0,
$$

over a $z$-domain that includes a turning point, and the construction of a related equation. Elucidations of this statement are the following:

(i) The differential equation (1.1) is one in which $\lambda$ is a parameter, real or complex, whose absolute value is large, namely $|\lambda|>N$ with some suitably large positive constant $N$. For generality, $\lambda$ will be dealt with as complex.

(ii) The variable $z$ ranges over a closed bounded domain, in which certain hypotheses, that will be enunciated, are fulfilled. It may be real or complex. For generality it will be dealt with as complex.

(iii) The coefficient functions $P(z, \lambda), Q(z, \lambda)$, and $R(z, \lambda)$ are power series in $1 / \lambda$, namely

$$
\begin{aligned}
& P(z, \lambda)=\sum_{n=0}^{\infty} \frac{P_{n}(z)}{\lambda^{n}}, \\
& Q(z, \lambda)=\sum_{n=0}^{\infty} \frac{Q_{n}(z)}{\lambda^{n}}, \\
& R(z, \lambda)=\sum_{n=0}^{\infty} \frac{R_{n}(z)}{\lambda^{n}},
\end{aligned}
$$

the series converging when $|\lambda|$ is sufficiently large, and their coefficients $P_{n}(z), Q_{n}(z)$ and $R_{n}(z)$ being analytic (indefinitely differentiable) over the $z$-domain.

(iv) The $z$-domain contains a (just one) zero of the function $P_{0}(z)$, this zero being of the first order. A zero of $P_{0}(z)$ is called a turning point of the differential equation, the order of the zero being the order of the turning point.

(v) A differential equation is said to be related to a given one if it has known solution forms, and its coefficients coincide with those of the given equation to the extent of all terms to a prescribed degree in $1 / \lambda$.

Received by the editors March 11, 1958.

(1) Sponsored by the United States Army under Contract No. DA-11-022-2059. 
A turning point is not, in the classical sense, a singular point of a differential equation. The solutions there are uniform. Nevertheless, the point is critical in a specific way for the asymptotic representations of the solutions. A differential equation (1.1) has associated with it the algebraic equation

$$
\chi^{4}+P_{0}(z) \chi^{2}=0
$$

which is known as its characteristic or auxiliary equation. The roots of this have an invariant coincidence pattern over any $z$-region that includes no turning point. Thus two roots, say $\chi_{1}(z)$ and $\chi_{2}(z) \equiv 0$, coincide identically, whereas $\chi_{2}(z)$ and the remaining two roots, $\chi_{3}(z) \equiv i P_{0}^{1 / 2}(z)$, and $\chi_{4}(z)$ $\equiv-i P_{0}^{1 / 2}(z)$, remain distinct. Where such invariance maintains, classical theory is applicable for the derivation of formal solutions, these being of the forms

$$
\exp \left(i \lambda \int \chi_{j}(z) d z\right) \sum_{n=0}^{\infty} \frac{\chi_{j, n}(z)}{\lambda^{n}}, \quad j=1,2,3,4,
$$

with analytic coefficients $\chi_{j, n}(z)$. At a turning point the coincidence pattern is abruptly different, all four roots being coincident there because they all vanish there. The effect of that is to engender singularities in at least some of the functions $\chi_{j, n}(z)$. The useableness of the expressions (1.4) is thereby destroyed.

A related equation has been found, in the case of many linear ordinary differential equations, to supply a medium through which the asymptotic solution forms can be deduced. Analytical method which is quite familiar is applicable to that end. The key to this deduction lies, of course, in the discoverability or constructibility of a related equation.

I submit this paper as a second study of the differential equation (1.1). In method, scope and objective it is in part parallel to, but also in large part divergent from, the earlier paper [1]. The analysis here is more direct and self-contained, and obviates what heretofore were substantial complications. The theory as here presented seems more perspicuous, and its scope is wider. In the paper [1] a certain distinctive sub-class of equations (1.1) could not be dealt with; the present paper fills this omission.

By contrast with the paper [1] the present one has been delimited to the formal derivations. The analysis by which the asymptotic solutions are inferred from the solutions of the related equation is omitted. The omission has been partly dictated by the desire to keep the dimensions of the paper within bounds, but partly also by the desire to avoid extensive repetition with the paper [1]. The latter, to be sure, is lacunary in the matter of equations (1.1) of the distinctive category. The analysis which establishes the asymptotic solutions of such equations remains to be given.

As in the paper [1], it is to be remarked that the "Orr-Sommerfeld" 
equation of hydrodynamics, namely

$$
\frac{d^{4} \psi}{d y^{4}}-2 \alpha^{2} \frac{d^{2} \psi}{d y^{2}}+\alpha^{4} \psi-i \alpha R\left[(\omega-c)\left(\frac{d^{2} \psi}{d y^{2}}-\alpha^{2} \psi\right)-\frac{d^{2} \omega}{d y^{2}}\right]=0,
$$

is of the type (1.1). The substitutions

$$
z=y-y_{0}, \quad \omega=\psi, \quad \lambda^{2}=i \alpha R\left[\frac{d \omega}{d y}\right]_{y=y_{0}},
$$

transform it into the form (1.1) with $Q(z, \lambda) \equiv 0$, and with

$$
\begin{array}{ll}
P_{0}(z)=\frac{\omega(y)-c}{\left[\frac{d \omega}{d y}\right]_{y=y_{0}}} ; \quad P_{2}=-2 \alpha^{2} ; P_{n} \equiv 0, & \text { for } n \neq 0,2 . \\
R_{0}(z)=-\alpha^{2} P_{0}(z)-P_{0}^{\prime \prime}(z) ; \quad R_{2}=\alpha^{4} ; R_{n} \equiv 0, & \text { for } n \neq 0,2 .
\end{array}
$$

The existing studies of equations of the type (1.1) have mainly been inspired by the equation (1.5). Papers by W. Wasow [2] and by C. C. Lin [3] are especially to be noted in this connection. These authors, as well as the paper $[1]$, give further references.

The present paper is self-contained. No reference to the paper [1] is called for. The matter which is critical to all the formal manipulations here developed is the avoidance of singularities at the turning point. It is of some interest to note that despite this stricture the derivations set forth are all performable in terms of quadratures when only a single solution of a certain differential equation of the second order, the equation (2.3) below, has been found.

2. Preliminaries. The differential equation (1.1) is unchanged in form by a translation of coördinates. We may, therefore, take the origin at the turning point, and that we shall do. In consequence of it, and because there is no other turning point,

$$
P_{0}(0)=0 ; \quad P_{0}(z) \neq 0, \quad \text { for } z \neq 0 .
$$

The parameter $\lambda$ is not identified by the equation (1.1), since it occurs in the equation only in products with other coefficients. A normalizing relation to inhibit the transfer of constant factors to or from $\lambda$ is needed. We shall, to that end, adopt the relation

$$
P_{0}^{\prime}(0)=1,
$$

observing this to be permissible under the hypothesis that has been made, that the turning point is of the first order.

The limiting form of the Equation (1.1) as $\lambda \rightarrow \infty$ is the differential equation of the second order 


$$
P_{0}(z) u^{\prime \prime}+Q_{0}(z) u^{\prime}+R_{0}(z) u=0 .
$$

The origin is a point at which the leading coefficient of this equation vanishes. It is therefore in general a regular singular point, though it is not such in the special cases in which the remaining coefficients also vanish there. However that may be, the equation has, relative to the origin, the exponents 0 and $\rho$, where, in accordance with the evaluation (2.2),

$$
\rho=1-Q_{0}(0) \text {. }
$$

We shall adopt it as a hypothesis that

$$
\rho \text { is not a negative integer }\left({ }^{2}\right) \text {. }
$$

In association with its exponent $\rho$ the differential equation (2.3) has a solution $u_{\rho}(z)$, whose quotient by $z^{\rho}$ is a power series in $z$ with the constant term 1. The formula

$$
u_{0}(z)=u_{\rho}(z) \int \frac{d z}{u_{\rho}^{2}(z) \theta(z)}
$$

in which

$$
\theta(z)=\exp \left(\int \frac{Q_{0}(z)}{P_{0}(z)} d z\right)
$$

familiarly gives a solution $u_{0}(z)$ which is linearly independent of $u_{\rho}(z)$, and is associated with the exponent 0 .

In general only one solution of the pair $u_{\rho}(z), u_{0}(z)$, is analytic at the origin, the other one having a branch point there. Thus when $\rho$ is not an integer, $u_{0}(z)$ is analytic, while $u_{\rho}$ is multiple valued like $z^{\rho}$, whereas, on the other hand, when $\rho$ is an integer, $u_{\rho}$ is analytic and $u_{0}(z)$ ordinarily of the character of $u_{\rho}(z) \log z$. There are, however, instances-ones in which $\rho$ is an integerin which the solutions $u_{\rho}(z)$ and $u_{0}(z)$ are both analytic. The equation (2.3) may, after all, not have any singularity.

It will be found requisite in the course of the discussion to restrict the $z$-domain from including any zero of $u_{\rho}(z)$ other than that at the origin. Also to require the exclusion of such zeros of the function $\int_{0}^{z} P_{0}^{1 / 2}(z) d z$, - a matter which is not implied by the condition (2.1) when $z$ or $P_{0}(z)$ is complex. We shall therefore adopt the hypotheses:

$$
u_{\rho}(z) \neq 0
$$

for $z \neq 0$,

and

(2) A conjecture is that this restriction could be replaced by an alternative one by appropriately rewriting the paper. However, as was remarked in the paper [1], adjoint equatiuns (1.1) have exponents with opposite signs. The conclusions for an equation with a negative integral $\rho$ are thus inferable from those of its adjoint. 


$$
\int_{0}^{z} P_{0}^{1 / 2}(z) d z \neq 0, \quad \text { for } z \neq 0 .
$$

It will be convenient to symbolize the left-ha nd member of the equation (1.1) by $\mathscr{L}(w)$, namely to set

$$
\mathscr{L}(w) \equiv w^{\prime \prime \prime \prime}+\lambda^{2}\left\{P(z, \lambda) w^{\prime \prime}+Q(z, \lambda) w^{\prime}+R(z, \lambda) w\right\} .
$$

PART I. FORMAL SOlutions AS POWER SERIES IN $1 / \lambda$

3. An algorithm. Let $w_{*}(\varepsilon)$ denote the power series in $1 / \lambda$,

$$
w_{*}(z)=\sum_{n=0}^{\infty} \frac{U_{n}(z)}{\lambda^{n}},
$$

in which the coefficients $U_{n}(z)$ are, for the moment, unspecified, but are presumed to be analytic. The substitution of this into the expression (2.7) yields formally the relation

$$
\mathcal{L}\left(w_{*}\right) \equiv \sum_{n=0}^{\infty} \lambda^{2-n}\left\{P_{0} U_{n}^{\prime \prime}+Q_{0} U_{n}^{\prime}+R_{0} U_{n}+h_{n}(U)\right\},
$$

in which

$$
\begin{aligned}
& h_{0}(U) \equiv 0, \\
& h_{1}(U) \equiv\left\{P_{1} U_{0}^{\prime \prime}+Q_{1} U_{0}^{\prime}+R_{1} U_{0}\right\}, \\
& h_{n}(U)=U_{n-2}^{\prime \prime \prime}+\sum_{j=1}^{n}\left\{P_{j} U_{n-j}^{\prime \prime}+Q_{j} U_{n-j}^{\prime}+R_{j} U_{n-j}\right\}, \\
& \qquad n=2,3,4, \cdots .
\end{aligned}
$$

The term in $\lambda^{2-n}$, for any $n$, can be made to vanish from the relation (3.2) by taking $U_{n}(z)$ to fulfill the equation

$$
P_{0} U_{n}^{\prime \prime}+Q_{0} U_{n}^{\prime}+R_{0} U_{n}=-h_{n}(U) .
$$

Since the expression $h_{n}(U)$ involves only those functions $U_{j}(z)$ for which $j<n$, these equations may be taken successively for $n=0,1,2, \cdots$, to determine the respective functions $U_{n}(z)$. By this means $\mathcal{L}\left(w_{*}\right)$, as a power series in $1 / \lambda$, is made to vanish term by term. The resulting series $w_{*}(z)$ is accordingly a formal solution of the equation $\mathfrak{L}(w)=0$, namely of the given differential equation (1.1). We use the adjective "formal" here to disclaim any assertion as to either the convergence of the series, or the legitimacy of the substitution process described. That the series obtained may indeed be divergent can easily be shown. In any instance in which $R(z, \lambda) \equiv 0$, for instance, the process described permits the choice of each function $U_{n}(z)$ as an arbitrary constant.

The equation (3.4) with $n=0$ is solved by the formula 


$$
U_{0}(z)=c_{0} u_{0}(z)+k_{0} u_{\rho}(z),
$$

in which $c_{0}$ and $k_{0}$ are arbitrary constants. That the equations for successive values of $n$ can be solved by quadratures is shown as follows. Suppose that for any $n$ the functions $U_{j}(z)$ with $j<n$ have been determined. The function $h_{n}(U)$ is then known. With $u$ signifying either $u_{0}(z)$ or $u_{\rho}(z)$ as may be convenient, the Equation (3.4) can be put into the form

$$
P_{0} u\left(\frac{U_{n}}{u}\right)^{\prime \prime}+\left\{2 P_{0} u^{\prime}+Q_{0} u\right\}\left(\frac{U_{n}}{u}\right)^{\prime}=-h_{n}(U) .
$$

It thus has the first integral

$$
u^{2} \theta\left(\frac{U_{n}}{u}\right)^{\prime}=H_{n}(U, u)
$$

in which

$$
H_{n}(U, u)=\int \frac{u \theta h_{n}(U)}{-P_{0}} d z .
$$

A further quadrature yields the formula

$$
U_{n}(z)=u(z) \int \frac{H_{n}(U, u)}{u^{2} \theta} d z+c_{n} u_{0}(z)+k_{n} u_{\rho}(z), \quad n=1,2,3, \cdots,
$$

$c_{n}$ and $k_{n}$ being constants of integration.

By the formulas (3.7), taken for successive $n$, the function $U_{n}(z)$ is made to depend upon $(2 n+2)$ constants of integration. For which, if any, of these constants $U_{n}(z)$ is analytic, namely devoid of a singularity at $z=0$, remains to be determined. That, it will be shown, depends upon whether the one or the other of the solutions $u_{\rho}(z), u_{0}(z)$, is analytic, or whether they are both so.

We observe that, by virtue of the evaluation (2.4), the function $\theta(z)$, whose formula is (2.6), is the product of $z^{1-\rho}$ by a factor that is analytic and nonvanishing at $z=0$.

4. Analyticity when $\rho$ is not an integer. When the exponent $\rho$ is nonintegral, the solution $u_{\rho}(z)$ is not analytic, but $u_{0}(z)$ is so. The formula (3.5) therefore gives an analytic determination of $U_{0}(z)$ if, and only if, $k_{0}=0$. Suppose, now, that for some $n$ analytic determinations of the functions $U_{j}(z)$ with $j<n$ have been possible, and have been made. The function $h_{n}(U)$ is therewith analytic, and by the formula (3.6), with $u_{0}(z)$ in the place of $u(z)$, the function $H_{n}\left(U, u_{0}\right)$ is, like $\theta(z)$, the product of $z^{1-\rho}$ by a power series in $z$. The formula (3.7) is thus seen to give an analytic determination of $U_{n}(z)$ provided $k_{n}=0$. The constants $c_{n}$ remain arbitrary.

To analyse the dependence of the functions $U_{n}(z)$ upon the constants $c_{n}$, the following lemma is useful. 
Lemma. If $\xi_{n}(z), n=0,1,2, \cdots$, is any sequence of analytic functions, and

$$
\Xi_{j}(z)=\sum_{i=0}^{j} c_{i} \xi_{j-i}(z)
$$

with constant coefficients $c_{i}$, then

$$
h_{n}(\Xi)=\sum_{i=0}^{n-1} c_{i} h_{n-i}(\xi)
$$

and by consequence

$$
H_{n}(\Xi, u)=\sum_{i=0}^{n-1} c_{\imath} H_{n-i}(\xi, u) .
$$

This can be proved as follows: By virtue of the relations (4.1) the formulas (3.3) give

$$
h_{n}(\Xi)=\sum_{i=0}^{n-2} c_{i} \xi_{n-2-i}^{\prime \prime \prime \prime}+\sum_{j=1}^{n} \sum_{i=0}^{n-j} c_{i}\left\{P_{j} \xi_{n-j-i}^{\prime \prime}+Q_{j} \xi_{n-j-i}^{\prime}+R_{j} \xi_{n-j-i}\right\} .
$$

An interchange of the order of summation yields the alternative form

$$
h_{n}(\Xi) \equiv \sum_{i=0}^{n-2} c_{i} \xi_{n-i-2}^{\prime \prime \prime \prime}+\sum_{i=0}^{n-1} c_{i} \sum_{j=1}^{n-i}\left\{P_{j} \xi_{n-i-j}^{\prime \prime}+Q_{j} \xi_{n-i-j}^{\prime}+R_{j} \xi_{n-i-j}\right\}
$$

and this is recognizable to be the relation (4.2). The conclusion (4.3) follows through the formula (3.6).

To apply this lemma, let the sequence of functions $\phi_{n}(z)$ be defined thus

$$
\begin{aligned}
\phi_{0}(z) & \equiv u_{0}(z), \\
\phi_{n}(z) & =u_{0}(z) \int \frac{H_{n}\left(\phi, u_{0}\right)}{u_{0}^{2} \theta} d z, \quad n=1,2,3, \ldots .
\end{aligned}
$$

These functions, it was observed above, are analytic. The relation

$$
U_{j}(z)=\sum_{i=0}^{i} c_{i} \phi_{j-i}(z),
$$

is valid when $j=0$. Suppose it to be valid when $j<n$. Then by the lemma

$$
H_{n}\left(U, u_{0}\right)=\sum_{i=0}^{n-1} c_{i} H_{n-i}\left(\phi, u_{0}\right)
$$

whereas by virtue of this the formula (3.7), with $k_{n}=0$, assumes the form (4.5) with $j=n$. The relations (4.5) are thus valid for all $j$, which is to say that an analytic formal solution (3.1) is of the form, and only of the form, 


$$
w_{*}(z)=\sum_{n=0}^{\infty} \frac{1}{\lambda^{n}} \sum_{i=0}^{n} c_{i} \phi_{n-i}(z) .
$$

This, however, is equivalently expressed as

$$
w_{*}(z)=c(\lambda) \sum_{n=0}^{\infty} \frac{\phi_{n}(z)}{\lambda^{n}},
$$

with

$$
c(\lambda)=\sum_{n=0}^{\infty} \frac{c_{n}}{\lambda^{n}} .
$$

The constants $c_{n}$ are thus seen to be involved only in a factor which is independent of $z$, in accordance with which formal solutions engendered by different choices of the constants are linearly dependent. A formulation of this conclusion is the following.

ThEOREM 1. A differential equation (1.1) whose limiting form (2.3) has a nonintegral exponent at the turning point admits the formal solution (3.1) in which the coefficients are the analytic functions $\phi_{n}(z)$. Every formal solution which is a power series in $1 / \lambda$ with coefficients that are analytic in $z$ is linearly dependent upon the one that is given by

$$
U_{n}(z) \equiv \phi_{n}(z) \text {. }
$$

5. Analyticity when $\rho$ is an integer and $u_{0}(z)$ is not analytic. When $\rho$ is an integer - a non-negative one by hypothesis - the solution $u_{\rho}(z)$ of the differential equation (2.3) is analytic. In general, then, $u_{0}(z)$ includes a nonvanishing multiple of $u_{\rho}(z) \log z$, and by virtue of that has a singularity at $z=0$. It is this case that we shall now consider.

As it is expressed in the form (3.5), the function $U_{0}(z)$ is analytic if, and only if, $c_{0}=0$. For positive $n$ the formula (3.7), with $u_{\rho}(z)$ in the place of $u(z)$, and with the use of the evaluation (2.5), can be written in the form

$$
U_{n}(z)=u_{\rho}(z) \int \frac{\left\{H_{n}\left(U, u_{\rho}\right)+c_{n}\right\}}{u_{\rho}^{2} \theta} d z+k_{n} u_{\rho}(z) .
$$

Suppose that for some $n$ analytic determinations of the functions $U_{j}(z)$ with $j<n$ have been possible and have been made. The functions $h_{n}(U)$ and $\left\{H_{n}\left(U, u_{\rho}\right)+c_{n}\right\}$ are therewith analytic, the latter, when expressed as a power series in $z$, having a constant term which depends linearly upon $c_{n}$. The factor $1 / u_{\nu}^{2} \theta$ is the product of $z^{-1-\rho}$ by a power series in $z$, and in this series the coefficient of $z^{p}$ is not zero, because if it were the solution $u_{0}(z)$ would be analytic. Therefore the integrand of the formula (5.1) has a residue which is linear in $c_{n}$, and which vanishes for a specific unique choice of $c_{n}$. With that choice the function $U_{n}(z)$ is seen from the formula (5.1) to be 
analytic, and to be so with an arbitrary constant $k_{n}$.

The dependence of $U_{n}(z)$ upon its constants can be analyzed as follows. Let the sequence of functions $\psi_{n}(z)$ be defined thus

$$
\begin{aligned}
& \psi_{0}(z) \equiv u_{\rho}(z), \\
& \psi_{n}(z)=u_{\rho}(z) \int \frac{\left\{H_{n}\left(\psi, u_{\rho}\right)-a_{n}\right\}}{u_{\rho}^{2} \theta} d z, \quad n=1,2,3, \cdots,
\end{aligned}
$$

the constants $a_{n}$ being those for which these functions are analytic. Therewith the relation

$$
U_{j}(z)=\sum_{i=0}^{3} k_{i} \psi_{j-i}(z),
$$

is valid when $j=0$. Suppose it to be valid when $j<n$. Then by the lemma of $\S 4$

$$
H_{n}\left(U, u_{\rho}\right)=\sum_{i=0}^{n-1} k_{i} H_{n-i}(\psi, u),
$$

whereas this implies through the formula (5.1) that

$$
U_{n}(z)=\sum_{i=0}^{n} k_{i} \psi_{n-i}(z)+\left\{c_{n}+\sum_{i=0}^{n-1} k_{i} a_{n-i}\right\} u_{0}(z) .
$$

A necessary and sufficient condition for the analyticity of $U_{n}(z)$ is thus that $c_{n}$ be chosen to fulfill the equation

$$
c_{n}+\sum_{i=0}^{n-1} k_{i} a_{n-i}=0 .
$$

With this choice the evaluation (5.4) reduces to (5.3) with $j=n$. With the constants $c_{n}$ chosen to fulfill the linear system of equations

$$
\begin{aligned}
c_{0} & =0, \\
c_{n} & =-\sum_{i=0}^{n-1} a_{n-i} k_{i},
\end{aligned}
$$

the formulas (5.3) are thus valid for all $j$, and the formal solution (3.1) with analytic coefficients is accordingly

$$
w_{*}(z)=\sum_{n=0}^{\infty} \frac{1}{\lambda^{n}} \sum_{i=0}^{n} k_{i} \psi_{n-i}(z) .
$$

This is equivalently expressed in the form

with

$$
w_{*}(z)=k(\lambda) \sum_{n=0}^{\infty} \frac{\psi_{n}(z)}{\lambda^{n}}
$$




$$
k(\lambda)=\sum_{n=0}^{\infty} \frac{k_{n}}{\lambda^{n}} .
$$

A formulation of this conclusion is the following:

Theorem 2. A differential equation (1.1) whose limiting form (2.3) has integral exponents but only a one-parameter family of analytic solutions admits the formal solution (3.1) in which the coefficients are the analytic functions $\psi_{n}(z)$. Every formal solution which is a power series in $1 / \lambda$ with coefficients that are analytic in $z$ is linearly dependent upon the one that is given by

$$
U_{n}(z) \equiv \psi_{n}(z) .
$$

6. Analyticity when $u_{\rho}(z)$ and $u_{0}(z)$ are both analytic. The differential equation (2.3) may have at $z=0$ only an apparent singularity or no singularity at all, the latter being the case when $Q_{0}(z)$ and $R_{0}(z)$ as well as $P_{0}(z)$ vanish there. When that is so-the instance cannot arise unless $\rho$ is a positive integer-the solutions $u_{\rho}(z)$ and $u_{0}(z)$ are both analytic. We now consider this case.

Let the function sequences $\Phi_{n}(z)$ and $\Psi_{n}(z)$ be defined by the formulas

$$
\begin{aligned}
& \Phi_{0}(z) \equiv u_{0}(z), \\
& \Phi_{n}(z)=u_{0}(z) \int \frac{\left\{H_{n}\left(\Phi, u_{0}\right)-a_{n} \log z\right\}}{u_{0}^{2} \theta} d z, \\
& \Psi_{0}(z) \equiv u_{\rho}(z), \\
& \Psi_{n}(z)=u_{0}(z) \int \frac{\left\{H_{n}\left(\Psi, u_{0}\right)-b_{n} \log z\right\}}{u_{0}^{2} \theta} d z, n=1,2,3, \cdots,
\end{aligned}
$$

the constants $a_{n}$ and $b_{n}$ being those for which these functions are analytic. That such constants exist and are uniquely defined can be shown inductively as follows.

The functions $\Phi_{j}(z)$ and $\Psi_{j}(z)$ are analytic when $j=0$. Suppose they are so when $j<n$. The functions $h_{n}(\Phi)$ and $h_{n}(\Psi)$ are then analytic, and the integrands of the formulas (3.6) for $H_{n}\left(\Phi, u_{0}\right)$ and $H_{n}\left(\Psi, u_{0}\right)$ accordingly each have a pole of the order $\rho$. With $a_{n}$ and $b_{n}$ determined as the respective residues at these poles, the integrands of the formulas (6.1) for $\Phi_{n}(z)$ and $\Psi_{n}(z)$ are analytic, and therewith $\Phi_{n}(z)$ and $\Psi_{n}(z)$, themselves, are analytic.

Consider, now, the relation

$$
U_{j}(z)=\sum_{i=0}^{j}\left\{c_{i} \Phi_{j-i}(z)+k_{i} \Psi_{j-i}(z)\right\} .
$$

It is valid, and gives an analytic determination with arbitrary constants $c_{0}$ and $k_{0}$, when $j=0$. Suppose it is valid when $j<n$. By the lemma of $\S 4$, then, 


$$
H_{n}\left(U, u_{0}\right)=\sum_{i=0}^{n-1}\left\{c_{i} H_{n-i}\left(\Phi, u_{0}\right)+k_{i} H_{n-i}\left(\Psi, u_{0}\right)\right\},
$$

and with this the formula (3.7), with $u_{0}(z)$ in the place of $u(z)$, assumes the form

$$
\begin{aligned}
U_{n}(z)= & \sum_{i=0}^{n-1}\left\{c_{i} \Phi_{n-i}(z)+k_{i} \Psi_{n-i}(z)\right\} \\
& +\left[\sum_{i=0}^{n-1}\left\{c_{i} a_{n-i}+k_{i} b_{n-i}\right\}\right] u_{0}(z) \int \frac{\log z}{u_{0}^{2} \theta} d z .
\end{aligned}
$$

This reduces to the relation (6.2) with $j=n$ if the constants $c_{i}$ and $k_{i}$ are chosen to fulfill the equation

$$
\sum_{i=0}^{n-1}\left\{a_{n-i} c_{i}+b_{n-i} k_{i}\right\}=0 .
$$

The relations (6.2) are assured for all $j$ if the equations (6.4) are fulfilled for $n=1,2,3, \cdots$.

The conclusions that are to be drawn from this are different according as the constants $a_{n}, b_{n}$, are, or are not, all zero. If $a_{n}=b_{n}=0$, for all $n$, the relations (6.4) are vacuous, and leave the constants $c_{i}$ and $k_{i}$ arbitrary. With a special choice of these constants the relations $(6.2)$ reduce to $U_{j}(z)=\Phi_{j}(z)$, and with another they reduce to $U_{j}(z)=\Psi_{j}(z)$. The conclusion which this permits may be formulated thus:

TheOREM 3. A differential equation (1.1) for which the functions of the sequences (6.1) with $a_{n}=b_{n}=0 ; n=0,1,2, \cdots$ are analytic, admits two linearly independent formal solutions which are power series in $1 / \lambda$ with coefficients that are analytic in $z$. Two such formal solutions are

$$
w_{*}(z)=\sum_{n=0}^{\infty} \frac{\Phi_{n}(z)}{\lambda^{n}}
$$

and

$$
w_{\rho, *}(z)=\sum_{n=0}^{\infty} \frac{\Psi_{n}(z)}{\lambda^{n}} .
$$

It can easily be shown by examples that differential equations of the type referred to in this theorem exist. Thus the equation

$$
w^{\prime \prime \prime \prime}+\lambda^{2}\left\{\left(2 z+\frac{z^{2}}{\lambda}-\frac{2 z+1}{\lambda^{2}}\right) w^{\prime \prime}-\left(2+\frac{2 z}{\lambda}-\frac{2}{\lambda^{2}}\right) w^{\prime}+\frac{2}{\lambda} w\right\}=0,
$$

whose limiting form (2.3) has an apparent singularity at $z=0$, admits the solutions 


$$
\begin{aligned}
& w_{*}(z)=1+\frac{z}{\lambda}-\frac{1}{\lambda^{2}}, \\
& w_{2, *}(z)=z^{2}+\frac{1}{\lambda} .
\end{aligned}
$$

The equation

$$
w^{\prime \prime \prime \prime}+\lambda^{2}\left\{\left(z-\frac{2}{\lambda^{2}}\right) w \omega^{\prime \prime}-\left(z-\frac{1}{\lambda^{2}}\right) w\right\}=0,
$$

whose limiting form (2.3) has no singularity, admits the solutions

$$
\begin{aligned}
w_{*}(z) & =\cosh z, \\
w_{1, *}(z) & =\sinh z .
\end{aligned}
$$

When the constants $a_{n}, b_{n}$ do not all vanish, an integer $N$ is specified by the relations

$$
\begin{aligned}
a_{n}=b_{n} & =0, & \text { for } n<N, \\
\left|a_{N}\right|+\left|b_{N}\right| & \neq 0 . &
\end{aligned}
$$

The Equations (6.4) are then subsumable into the relation

$$
a(\lambda) c(\lambda)+b(\lambda) k(\lambda)=0,
$$

in which $c(\lambda)$ and $k(\lambda)$ have respectively the formulas (4.7) and (5.6), and

$$
a(\lambda)=\sum_{n=0}^{\infty} \frac{a_{N+n}}{\lambda^{n}}, \quad b(\lambda)=\sum_{n=0}^{\infty} \frac{b_{N+n}}{\lambda^{n}} .
$$

In accordance with this a particular permissible choice of the constants $c_{n}$ and $k_{n}$ is that for which $k(\lambda)=a(\lambda)$, and $c(\lambda)=-b(\lambda)$, and every choice must be proportional to this particular one. With the particular choice the formal solution (3.1), (6.2) is

$$
w(z)=\sum_{n=0}^{\infty} \frac{1}{\lambda^{n}} \sum_{j=0}^{n}\left\{a_{N+j} \Psi_{n-j}(z)-b_{N+j} \Phi_{n-j}(z)\right\} .
$$

Our conclusion is the following one.

THEOREM 4. A differential equation (1.1) for which the functions of the sequences (6.1) are analytic with constants $a_{n}, b_{n}$ that fulfill the relations (6.7), admits the formal solution (6.8). Every formal solution which is a power series in $1 / \lambda$ with coefficients that are analytic in $z$ is linearly dependent upon the solution (6.8).

That differential equations of the type referred to in this theorem exist is easily shown. The equations 


$$
\begin{aligned}
w^{\prime \prime \prime \prime}+\lambda^{2}\left\{\left(z+\frac{z}{\lambda^{2}}\right) w^{\prime \prime}-w^{\prime}\right\} & =0, \\
w^{\prime \prime \prime \prime}+\lambda^{2}\left\{z w^{\prime \prime}+\frac{1}{\lambda} w^{\prime}\right\} & =0,
\end{aligned}
$$

have limiting forms (2.3) which have respectively an apparent singularity and no singularity at $z=0$. They each admit as formal solutions only those power series in $1 / \lambda$ in which the coefficients are constants. In each case, therefore, every formal solution of the type in question is linearly dependent upon the particular one $w_{*}(z) \equiv 1$.

PART II. THE DERIVATION OF FORMAL SOlUtions FROM A DIFFERENTIAL EQUATION OF THE THIRD ORDER

7. A formalism. The differential equation

$$
\dddot{v}+\lambda^{2} x \dot{v}-\lambda^{2} \mu v=0,
$$

in which $\mu$ is a parameter, and each superscribed dot signifies a differentiation with respect to $x$, is one whose solutions, as functions of $x, \lambda$ and $\mu$, are of known forms [4]. We propose to bank upon these solutions with $x$ functionally dependent upon $z$. The formula for this dependence we find it convenient to take in the form

$$
x=\int_{0}^{z} \sigma(z) d z,
$$

with an integrand $\sigma(z)$ which is analytic, and which, beyond that, is to be determined.

With $\nu$ an undetermined parameter, possibly a function of $\lambda$, and with $A(z, \nu), B(z, \nu)$ and $C(z, \nu)$ as undetermined multipliers that are dependent upon $\nu$, are analytic in $z$, and (although the notation does not indicate it) are functions of $\lambda$, the formula

$$
w(z, \mu)=A(z, \nu) v(x, \mu)+\frac{1}{\lambda} B(z, \nu) \dot{v}(x, \mu)+\frac{1}{\lambda^{2}} C(z, \nu) \ddot{v}(x, \mu)
$$

formally relates an expression $w(z, \mu)$ with each solution $v(x, \mu)$ of the equation (7.1). The differentiation of this with respect to $z$, followed by the elimination of $\dddot{v}(x, \mu)$ by use of the equation (7.1), yields the evaluation

$$
\begin{aligned}
w^{\prime}(z, \mu)= & {\left[A^{\prime}+\sigma \mu C\right] v+\left[\sigma(A-x C)+\frac{1}{\lambda} B^{\prime}\right] \dot{v} } \\
& +\left[\frac{1}{\lambda} \sigma B+\frac{1}{\lambda^{2}} C^{\prime}\right] \ddot{v} .
\end{aligned}
$$


The higher derivatives, as they are obtained successively by this process of differentiating and eliminating, are found to be

$$
w^{\prime \prime}(z, \mu)=\lambda H(\nu, \mu) v+\lambda J(\nu, \mu) \dot{v}+K(\nu) \ddot{v},
$$

and

(7.6) $w^{\prime \prime \prime}(z, \mu)=\left[\lambda^{2} \sigma \mu K+\lambda H^{\prime}\right] v+\left[-\lambda^{2} x \sigma K+\lambda\left(\sigma H+J^{\prime}\right)\right] \dot{v}+\left[\lambda \sigma J+K^{\prime}\right] \ddot{v}$, wherein $H, J$ and $K$ are given by the formulas

$$
\begin{aligned}
H(\nu, \mu)= & \sigma^{2} \mu B+\lambda^{-1}\left[A^{\prime \prime}+2 \sigma \mu C^{\prime}+\sigma^{\prime} \mu C\right], \\
J(\nu, \mu)= & -x \sigma^{2} B+\lambda^{-1}\left[2 \sigma A^{\prime}+\sigma^{\prime} A-2 x \sigma C^{\prime}-x \sigma^{\prime} C+(\mu-1) \sigma^{2} C\right] \\
& +\lambda^{-2} B^{\prime \prime}, \\
K(\nu)= & \sigma^{2}(A-x C)+\lambda^{-1}\left[2 \sigma B^{\prime}+\sigma^{\prime} B\right]+\lambda^{-2} C^{\prime \prime} .
\end{aligned}
$$

These expressions (7.7) depend upon $z$ and $\lambda$, of course, as well as upon the indicated parameters $\nu$ and $\mu$. One further differentiation gives

$$
\begin{aligned}
& w^{\prime \prime \prime \prime}(z, \mu)=\left[\lambda^{3} \sigma^{2} \mu J+\lambda^{2}\left(2 \sigma \mu K^{\prime}+\sigma^{\prime} \mu K\right)+\lambda H^{\prime \prime}\right] v \\
& \quad+\left[-\lambda^{3} x \sigma^{2} J+\lambda^{2}\left(\sigma^{2} \mu K-2 x \sigma K^{\prime}-x \sigma^{\prime} K-\sigma^{2} K\right)+\lambda\left(2 \sigma H^{\prime}+\sigma^{\prime} H+J^{\prime \prime}\right)\right] \dot{v} \\
& \quad+\left[\lambda^{2} x \sigma^{2} K+\lambda\left(\sigma^{2} H+2 \sigma J^{\prime}+\sigma^{\prime} J\right)+K^{\prime \prime}\right] \ddot{v},
\end{aligned}
$$

in consequence of which $\mathscr{L}(w)$, as given in $(2.7)$, is found to have the formula

$$
\mathcal{L}(w(z, \mu))=\lambda^{2} S_{1}(\nu, \mu) v(x, \mu)+\lambda^{2} S_{2}(\nu, \mu) \dot{v}(x, \mu)+\lambda S_{3}(\nu, \mu) \ddot{v}(x, \mu)
$$

with

$$
\begin{aligned}
S_{1}(\nu, \mu)= & \lambda\left[P H+\sigma^{2} \mu J\right]+\left[Q A^{\prime}+R A+\mu\left(\sigma Q C+2 \sigma K^{\prime}+\sigma^{\prime} K\right)\right] \\
& +\lambda^{-1} H^{\prime \prime}, \\
S_{2}(\nu, \mu)= & \lambda\left(P-x \sigma^{2}\right) J+\left[\sigma Q(A-x C)-2 x \sigma K^{\prime}-x \sigma^{\prime} K+(\mu-1) \sigma^{2} K\right] \\
& +\lambda^{-1}\left[Q B^{\prime}+R B+2 \sigma H^{\prime}+\sigma^{\prime} H+J^{\prime \prime}\right], \\
S_{3}(\nu, \mu)= & \lambda\left(P-x \sigma^{2}\right) K+\left[\sigma Q B+\sigma^{2} H+2 \sigma J^{\prime}+\sigma^{\prime} J\right] \\
& +\lambda^{-1}\left[Q C^{\prime}+R C+K^{\prime \prime}\right] .
\end{aligned}
$$

It is convenient to combine the relations (7.3)-(7.6) into a single one of vectorial form, namely

$$
\left[\begin{array}{c}
w(z, \mu) \\
w^{\prime}(z, \mu) \\
w^{\prime \prime}(z, \mu) \\
w^{\prime \prime \prime}(z, \mu)
\end{array}\right]=M(\nu, \mu)\left[\begin{array}{l}
v(x, \mu) \\
\dot{v}(x, \mu) \\
\ddot{v}(x, \mu)
\end{array}\right],
$$

by introducing the matrix 
(7.11) $\quad M(\nu, \mu)=\left[\begin{array}{lll}A(z, \nu) & \lambda^{-1} B(z, \nu) & \lambda^{-2} C(z, \nu) \\ A^{\prime}+\sigma \mu C & \sigma(A-x C)+\lambda^{-1} B^{\prime} & \lambda^{-1} \sigma B+\lambda^{-2} C^{\prime} \\ \lambda H(\nu, \mu) & \lambda J(\nu, \mu) & K(\nu) \\ \lambda^{2} \sigma K+\lambda H^{\prime} & -\lambda^{2} x \sigma K+\lambda\left(\sigma H+J^{\prime}\right) & \lambda \sigma J+K^{\prime}\end{array}\right]$.

Let $\nu, A, B$ and $C$ be taken, now, to be formal power series in $1 / \lambda$, namely,

$$
\nu=\sum_{n=0}^{\infty} \frac{\nu_{n}}{\lambda^{n}},
$$

and

$$
\begin{aligned}
& A(z, \nu)=\sum_{n=0}^{\infty} \frac{\alpha_{n}(z)}{\lambda^{n}}, \\
& B(z, \nu)=\sum_{n=0}^{\infty} \frac{\beta_{n}(z)}{\lambda^{n}}, \\
& C(z, \nu)=\sum_{n=0}^{\infty} \frac{\gamma_{n}(z)}{\lambda^{n}} .
\end{aligned}
$$

Our proximate concern will be with the formulas (7.8) and (7.9) when $\mu$ is equal to $\nu$, namely, more specifically, with the determination of the elements that have thus far been left unspecified so as to fulfill the formal relations

$$
S_{j}(\nu, \nu)=0,
$$$$
j=1,2,3 \text {. }
$$

By virtue of the formulas (7.12) and (7.13) the left-hand members of the Equations (7.14) are each expressible as the sum of a term in $\lambda$ and a formal power series in $1 / \lambda$. By reducing these expressions to zero term by term, the right-hand member of the relation (7.8) is made to vanish. In accordance with that the expression $w(z, \nu)$, or, as we shall more concisely write it, $w(z)$, which has the formulas

$$
\left[\begin{array}{c}
w(z) \\
w^{\prime}(z) \\
w^{\prime \prime}(z) \\
w^{\prime \prime \prime}(z)
\end{array}\right]=M(\nu, \nu)\left[\begin{array}{l}
v(x, \nu) \\
\dot{v}(x, \nu) \\
\ddot{v}(x, \nu)
\end{array}\right]
$$

will be a formal solution of the differential equation (1.1).

8. The determinations of $x$ and $\sigma(z)$. The terms in $\lambda$ in the left-hand members of the equations (7.14) are respectively

$$
\begin{aligned}
& \lambda \sigma^{2} \nu_{0}\left(P_{0}-x \sigma^{2}\right) \beta_{0}, \\
& \lambda \sigma^{2}\left(P_{0}-x \sigma^{2}\right) \beta_{0}, \\
& \lambda \sigma^{2}\left(P_{0}-x \sigma^{2}\right)\left(\alpha_{0}-x \gamma_{0}\right) .
\end{aligned}
$$


They vanish if $x$ is such as to fulfill the equation

$$
x \sigma^{2}=P_{0} .
$$

The square root of this equation, multiplied by $d z$, can, by virtue of the formula (7.2), be written as

$$
x^{1 / 2} d x=P_{0}^{1 / 2} d z
$$

this equation being equivalent to (8.1) if the $z$-domain and the complex $x$ plane are taken to be two-sheeted Riemann surfaces, each with a branch point at the origin. The equation

$$
x^{3}=\left\{\frac{3}{2} \int_{0}^{z} P_{0}^{1 / 2} d z\right\}^{2},
$$

which is thus obtainable by an integration, permits the determination of $x$ as an analytic function of $z$, because $P_{0}^{1 / 2}(z)$ has the character of $z^{1 / 2}$ near $z=0$. We may, therefore, write

$$
x=\left\{\frac{3}{2} \int_{0}^{z} P_{0}^{1 / 2} d z\right\}^{2 / 3} .
$$

As a function of $z$, therefore, $x$ has a simple zero at $z=0$. It has no other zero in the $z$-domain, by consequence of a hypothesis which was adopted in $\$ 2$.

The relation

$$
\sigma^{2}(z)=\frac{P_{0}(z)}{\left\{\frac{3}{2} \int_{0}^{2} P_{0}^{1 / 2} d z\right\}^{2 / 3}},
$$

which is consequent to (8.1) and (8.2), is analytic except for a removable singularity at $z=0$, and gives for $\sigma^{2}(z)$ a value which is everywhere different from zero. It permits a determination of $\sigma(z)$ which is analytic and nonvanishing over the $z$-domain, with

$$
\sigma(0)=1 .
$$

The mapping from $z$ to $x$ is accordingly conformal, and $z$ is, inversely, an analytic function of $x$.

By the determinations (8.2) and (8.3), the terms in $\lambda$ are removed from the expressions on the left of the equations (7.14), and these expressions are thus reduced to be formal power series in $1 / \lambda$.

9. The determinations of $\nu_{0}, \alpha_{0}(z), \beta_{0}(z)$, and $\gamma_{0}(z)$. The expressions $S_{j}(\nu, \nu)$ as power series in $1 / \lambda$, are found to have as their leading terms $S_{j}^{(0)}$ the functions of $\nu_{0}, \alpha_{0}, \beta_{0}$ and $\gamma_{0}$ with the formulas 


$$
\begin{aligned}
S_{1}^{(0)}\left(\nu_{0}, \alpha_{0}, \beta_{0}, \gamma_{0}\right)= & P_{0} \alpha_{0}^{\prime \prime}+\left[Q_{0}-4 \nu_{0} \sigma^{3}\right] \alpha_{0}^{\prime}+\left[R_{0}+6 \nu_{0} \sigma^{2} \sigma^{\prime}\right] \alpha_{0} \\
& +\nu_{0} \sigma^{2} P_{1} \beta_{0}-2 \nu_{0} \sigma P_{0} \gamma_{0}^{\prime}-\nu_{0}\left[T+2 \sigma^{4}\right] \gamma_{0}, \\
S_{2}^{(0)}\left(\nu_{0}, \alpha_{0}, \beta_{0}, \gamma_{0}\right)= & -2 \sigma P_{0} \alpha_{0}^{\prime}-T \alpha_{0}-P_{0} P_{1} \beta_{0}+2 x \sigma P_{0} \gamma_{0}^{\prime} \\
& +x\left[T+2 \sigma^{4}\right] \gamma_{0}, \\
S_{3}^{(0)}\left(\nu_{0}, \alpha_{0}, \beta_{0}, \gamma_{0}\right)= & \sigma^{2} P_{1} \alpha_{0}-2 \sigma P_{0} \beta_{0}^{\prime}-\left[T+\sigma^{4}\right] \beta_{0}-P_{0} P_{1} \gamma_{0},
\end{aligned}
$$

in which

$$
T=5 \sigma^{\prime} P_{0}-\sigma Q_{0}+\left(1-\nu_{0}\right) \sigma^{4} .
$$

These terms can, therefore, be made to vanish by choosing the elements $\nu_{0}, \alpha_{0}(z), \beta_{0}(z), \gamma_{0}(z)$, to fulfill the equations

$$
S_{j}^{(0)}\left(\nu_{0}, \alpha_{0}, \beta_{0}, \gamma_{0}\right)=0, \quad j=1,2,3 .
$$

We shall show that such a choice is possible, and-what is important-under the stipulation that $\alpha_{0}(z), \beta_{0}(z)$ and $\gamma_{0}(z)$ be analytic in $z$.

The system of equations (9.3) implies, and is implied by, the alternative one

$$
\begin{aligned}
P_{0}(z) S_{1}^{(0)}\left(\nu_{0}, \alpha_{0}, \beta_{0}, \gamma_{0}\right)+\nu_{0} \sigma^{2}(z) S_{2}^{(0)}\left(\nu_{0}, \alpha_{0}, \beta_{0}, \gamma_{0}\right) & =0, \\
S_{2}^{(0)}\left(\nu_{0}, \alpha_{0}, \beta_{0}, \gamma_{0}\right)+i x^{1 / 2} S_{3}^{(0)}\left(\nu_{0}, \alpha_{0}, \beta_{0}, \gamma_{0}\right) & =0, \\
S_{2}^{(0)}\left(\nu_{0}, \alpha_{0}, \beta_{0}, \gamma_{0}\right)-i x^{1 / 2} S_{3}^{(0)}\left(\nu_{0}, \alpha_{0}, \beta_{0}, \gamma_{0}\right) & =0 .
\end{aligned}
$$

By the use of the relation (8.1), the first equation (9.4) is found to be explicitly

$$
P_{0}^{2} \alpha_{0}^{\prime \prime}+P_{0}\left[Q_{0}+2 \nu_{0} \sigma^{3}\right] \alpha_{0}^{\prime}+\left[P_{0} R_{0}+6 \nu_{0} \sigma^{2} \sigma^{\prime} P_{0}-\nu_{0} \sigma^{2} T\right] \alpha_{0}=0 .
$$

It is thus free of the functions $\beta_{0}(z)$ and $\gamma_{0}(z)$, namely, is an ordinary differential equation in $\alpha_{0}(z)$. The substitution $\alpha_{0}=x^{-\rho_{u l}}$ transforms it into

$$
\begin{aligned}
& x^{2} P_{0}\left\{P_{0} u^{\prime \prime}+Q_{0} u^{\prime}+R_{0} u\right\} \\
& +\left(\nu_{0}-\rho\right) P_{0}\left\{2 x \sigma P_{0} u^{\prime}+\left[x \sigma^{\prime} P_{0}+x \sigma Q_{0}+\left(\nu_{0}-\rho-1\right) \sigma^{2} P_{0}\right] u\right\}=0 .
\end{aligned}
$$

Hence it is fulfilled by the determination

$$
\nu_{0}=\rho,
$$

and the choice of $u$ as a solution of the differential equation (2.3). This latter equation has the solution $u_{\rho}(z)$, which has the character of $z^{\rho}$ near $z=0$. Since $x^{\rho}$ also has that character, and is nonvanishing elsewhere in the $z$-domain, the determination

$$
\alpha_{0}(z)=\frac{u_{\rho}(z)}{x^{\rho}}
$$


is analytic, and, with (9.5), fulfills the first equation (9.4). In accordance with it

$$
\alpha_{0}(0)=1 .
$$

That $\alpha_{0}(z)$ is different from zero over the $z$-domain, follows from the relevant hypothesis imposed upon the domain with respect to the solution $u_{\rho}(z)$ in $\S 2$.

The second and third equations (9.4) are found, again by the use of the relation $(8.1)$, to be expressible as

$$
-2 \sigma P_{0}\left\{\alpha_{0}-x \gamma_{0} \pm i x^{1 / 2} \beta_{0}\right\}^{\prime}-\left[T \mp i \sigma P_{0}^{1 / 2} P_{1}\right]\left\{\alpha_{0}-x \gamma_{0} \pm i x^{1 / 2} \beta_{0}\right\}=0 .
$$

They are thus amenable to integrations as linear equations of the first order. The integrals are respectively

$$
\alpha_{0}-x \gamma_{0} \pm i x^{1 / 2} \beta_{0}=e^{\tau(z) \pm \zeta(z)},
$$

with

$$
\begin{aligned}
& \tau(z)=-\int_{0}^{z} \frac{T}{2 \sigma P_{0}} d z, \\
& \zeta(z)=\int_{0}^{z} \frac{P_{1}}{2 P_{0}^{1 / 2}} d z .
\end{aligned}
$$

The functions (9.9) exist, since the function $T$, as given by the formula (9.2), has a zero at $z=0$, by virtue of the determinations (9.5) and (2.4).

Combinations of the results (9.8) yield the evaluations

$$
\beta_{0}(z)=e^{\tau(z)} \frac{\sin \zeta(z)}{x^{1 / 2}}, \quad \gamma_{0}(z)=\frac{1}{x}\left\{\alpha_{0}(z)-e^{\tau(z)} \cos \zeta(z)\right\} .
$$

The singularities which appear here at $z=0$ are removable; the determinations accordingly analytic. The equation

$$
\left(\alpha_{0}-x \gamma_{0}\right)^{2}+x \beta_{0}^{2}=e^{2 \tau(z)},
$$

which follows from (9.10), establishes the fact that the quantity upon its left is nonvanishing over the $z$-domain. That fact will later have to be appealed to.

10. The determinations of $\nu_{n}, \alpha_{n}(z), \beta_{n}(z)$, and $\gamma_{n}(z)$. We shall suppose, now, that with an assigned $n$ analytic determinations of the elements $\nu_{k}$, $\alpha_{k}(z), \beta_{k}(z)$ and $\gamma_{k}(z)$ with $k<n$, to remove the terms in $1 / \lambda^{k}$ from the expressions $S_{j}(\nu, \nu)$, have been possible, and have been made, and shall show that as a consequence the same is possible for $k=n$.

By the collection of all relevant terms in the formulas (7.9) with $\nu$ in the place of $\mu$, the total coefficients of $1 / \lambda^{n}$ in the expressions $S_{j}(\nu, \nu)$ are found to be the left-hand members of equations 


$$
S_{j}^{(0)}\left(\rho, \alpha_{n}, \beta_{n}, \gamma_{n}\right)-f_{j, n}\left(z, \nu_{n}\right)=0, \quad j=1,2,3,
$$

in which the terms $S_{j}^{(0)}$ have the formulas (9.1) and the functions $f_{j, n}$ are specific analytic combinations of $\nu_{n}$ and elements that have already been determined. The dependence of the second one of these functions, namely, $f_{2, n}$, upon $\nu_{n}$, is of immediate moment. That is found to lodge exclusively in the term

$$
\sigma^{4}\left(\alpha_{0}-x \gamma_{0}\right) \nu_{n}
$$

which reduces, at $z=0$, to $\nu_{n}$ itself. With an appropriate determination of $\nu_{n}$ the function $f_{2, n}(z, \nu)$ accordingly vanishes at $z=0$. We shall adopt this determination, whereby it is assured that

$$
f_{2, n}\left(0, \nu_{n}\right)=0 .
$$

As a consequence, $f_{2, n}\left(z, \nu_{n}\right) / x$ is analytic. The equations (10.1) now constitute a nonhomogeneous differential system for the functions $\alpha_{n}, \beta_{n}(z)$, and $\gamma_{n}(z)$. An equivalent is the system

$$
\begin{gathered}
P_{0} S_{1}^{(0)}\left(\rho, \alpha_{n}, \beta_{n}, \gamma_{n}\right)+\rho \sigma^{2} S_{2}^{(0)}\left(\rho, \alpha_{n}, \beta_{n}, \gamma_{n}\right)=P_{0} f_{1, n}+\rho \sigma^{2} f_{2, n} \\
S_{2}^{(0)}\left(\rho, \alpha_{n}, \beta_{n}, \gamma_{n}\right)+i x^{1 / 2} S_{3}^{(0)}\left(\rho, \alpha_{n}, \beta_{n}, \gamma_{n}\right)=f_{2, n}+i x^{1 / 2} f_{3, n}, \\
S_{2}^{(0)}\left(\rho, \alpha_{n}, \beta_{n}, \gamma_{n}\right)-i x^{1 / 2} S_{3}^{(0)}\left(\rho, \alpha_{n}, \beta_{n}, \gamma_{n}\right)=f_{2, n}-i x^{1 / 2} f_{3, n} .
\end{gathered}
$$

The first equation (10.3) is, more explicitly,

$$
P_{0}^{2} \alpha_{n}^{\prime \prime}+P_{0}\left[Q_{0}+2 \rho \sigma^{3}\right] \alpha_{n}^{\prime}+\left[P_{0} R_{0}+6 \rho \sigma^{2} \sigma^{\prime} P_{0}-\rho \sigma^{2} T\right] \alpha_{n}=P_{0} f_{1, n}+\rho \sigma^{2} f_{2, n} .
$$

The substitution $\alpha_{n}=x^{-\rho} u_{n}$, transforms it into

$$
P_{0} u_{n}^{\prime \prime}+Q_{0} u_{n}^{\prime}+R_{n} u_{n}=x^{\rho}\left[f_{1, n}+\frac{\rho f_{2, n}}{x}\right] \text {. }
$$

This equation is of the type (3.4), and can be integrated in the manner of $\$ 3$. The determination of $\alpha_{n}(z)$ which is so obtained, namely,

$$
\alpha_{n}(z)=\alpha_{0}(z) \int_{0}^{z} \frac{1}{\theta u_{\rho}^{2}} \int_{0}^{z} x^{\rho}\left[f_{1, n}+\frac{\rho f_{2, n}}{x}\right] \frac{\theta u_{\rho}}{P_{0}} d z d z,
$$

is analytic. Moreover

$$
\alpha_{n}(0)=0 .
$$

The second and third equations (10.3) are expressible in the form

$$
\begin{aligned}
-2 \sigma P_{0}\left\{\alpha_{n}-x \gamma_{n} \pm i x^{1 / 2} \beta_{n}\right\}^{\prime}-\left[T \mp i \sigma P_{0}^{1 / 2} P_{1}\right]\left\{\alpha_{n}-x \gamma_{n} \pm i x^{1 / 2} \beta_{n}\right\} \\
=f_{2, n} \pm i x^{1 / 2} f_{3, n} .
\end{aligned}
$$

They have the integrals 


$$
\alpha_{n}-x \gamma_{n} \pm i \cdot x^{1 / 2} \beta_{n}=\int_{0}^{z}\left\{\frac{f_{2, n}(t)}{x(t)} \pm i \frac{f_{3 n}(t)}{x^{1 / 2}(t)}\right\} \frac{e^{\tau(z, t) \pm i \zeta(z, t)}}{2 \sigma^{3}(t)} d t
$$

in which

$$
\begin{aligned}
& \tau(z, t)=\tau(z)-\tau(t) \\
& \zeta(z, t)=\zeta(z)-\zeta(t)
\end{aligned}
$$

Combinations of these yield the analytic determinations

$$
\beta_{n}(z)=\frac{1}{x^{1 / 2}} \int_{0}^{z}\left\{\frac{f_{2, n}(t)}{x(t)} \sin \zeta(z, t)+\frac{f_{3, n}(l)}{x^{1 / 2}(t)} \cos \zeta(z, t)\right\} \frac{e^{\tau(z, t)}}{2 \sigma^{3}(t)} d t
$$

$$
\begin{aligned}
\gamma_{n}(z)=\frac{1}{x}\left[\alpha_{n}(z)-\int_{0}^{z}\left\{\frac{f_{2, n}(t)}{x(t)} \cos \zeta(z, l)\right.\right. & \\
& \left.\left.-\frac{f_{3, n}(t)}{x^{1 / 2}(t)} \sin \zeta(z, t)\right\} \frac{e^{\tau(z, t)}}{2 \sigma^{3}(t)} d t\right] .
\end{aligned}
$$

11. The functions $v_{q, j}(x, \mu)$. The solutions of the differential equation (7.1) have known forms $\left({ }^{3}\right)$ [4]. They can be expressed either as power series in $x$-more precisely in $\lambda^{2 / 3} x$-or asymptotically with respect to $\left|\lambda x^{3 / 2}\right|$. A selection of certain ones of these solutions, or rather of sets of three of them, is advantageously based upon their asymptotic forms. The domains in which specific forms are maintained are identifiable by the integers $q$ (positive, negative and zero), as is set forth below. An accepted notation for the respective solutions is $\left({ }^{4}\right) v_{q, j}(x, \mu), j=1,2,3$.

With

$$
X_{q}=\frac{1}{3} \exp \left[-(2 q+1) \frac{\pi i}{2}\right] \lambda x^{3 / 2}
$$

and with $\epsilon_{0}$ as an arbitrarily small positive constant, the forms are the following:

For

$$
\begin{aligned}
(q-3) \pi+\epsilon_{0} & \leqq \arg \left(\lambda x^{3 / 2}\right) \leqq q \pi-\epsilon_{0}, \\
v_{q, 1}(x, \mu) & \sim\left\{\frac{e^{(q+1) \pi i}}{X_{q}}\right\}^{\mu / 3+1 / 2} e^{-2 X_{q}} \sum_{n=0}^{\infty} \frac{\delta_{n}(\mu)}{\left(3 i X_{q}\right)^{n}},
\end{aligned}
$$

$\left.{ }^{3}\right)$ The formulas of the paper [4] are adaptable to the present equation (7.1) by replacing $\mu$ by $-\mu / 3$. Errata to be noted in [4] are: in formula (8.4) for $v_{q, 0}$ the factor $\exp (1-2 q) \pi i$ should be $\exp (1-2 q) \mu \pi i$; in formulas $(8.5)$ for $v_{q, 1}$ and $v_{q, 2}$, factors $(-1)^{n}$ should be inserted.

(4) The solution here denoted by $v_{q, 3}(x, \mu)$ is the solution $(i \lambda / 3)^{2 \mu} v_{0}$ of the paper [4], as that is adapted to the present equation (7.1) by the replacement of $\mu$ by $-\mu / 3$. 
and for

$$
\begin{aligned}
(q-2) \pi+\epsilon_{0} & \leqq \arg \left(\lambda x^{3 / 2}\right) \leqq(q+1) \pi-\epsilon_{0}, \\
v_{q, 2}(x, \mu) & \sim\left\{\frac{e^{(q+1) \pi i}}{X_{q}}\right\}^{\mu / 3+1 / 2} e^{2 X_{q}} \sum_{n=0}^{\infty} \frac{\delta_{n}(\mu)}{\left(-3 i X_{q}\right)^{n}},
\end{aligned}
$$

the coefficients $\delta_{n}(\mu)$ being obtainable from the recurrence formulas

$$
\begin{aligned}
& \delta_{0}= 1, \\
& \delta_{1}= \frac{i}{48}[4-3(5+2 \mu)(3+2 \mu)], \\
& \delta_{n}= \frac{i}{48 n}[4-3(6 n-1+2 \mu)(6 n-3+2 \mu)] \\
&+\frac{1}{192 n}(6 n-1+2 \mu)(6 n-5+2 \mu)(6 n-9+2 \mu) \delta_{n-2}, \\
& n=2,3,4, \cdots .
\end{aligned}
$$

For

$$
\begin{aligned}
(q-2) \pi+\epsilon_{0} & \leqq \arg \left(\lambda x^{3 / 2}\right) \leqq q \pi-\epsilon_{0} \\
v_{q, 3}(x, \mu) & \sim x^{\mu} \sum_{n=0}^{\infty} \frac{\kappa_{n}(\mu)}{\left(\lambda^{2} x^{3}\right)^{n}}
\end{aligned}
$$

with the coefficients

$$
\kappa_{n}(\mu)=\frac{\Gamma(1+\mu)}{3^{n} \Gamma(1+\mu-3 n) \Gamma(n+1)} .
$$

These solutions have the convergent formulas

$$
\begin{aligned}
v_{q, 1}(x, \mu)= & 2\left(\frac{\pi}{3}\right)^{1 / 2} \exp \left[(1-q)\left(\frac{\mu}{3}+\frac{1}{2}\right) \pi i\right] \\
& \cdot \sum_{n=0}^{\infty} \frac{\left(3 e^{(\pi i) / 3} X_{q}^{2 / 3}\right)^{n}}{\Gamma\left(1+\frac{\mu-n}{3}\right) \Gamma(n+1)}, \\
v_{q, 2}(x, \mu)= & 2\left(\frac{\pi}{3}\right)^{1 / 2} \exp \left[-q\left(\frac{\mu}{3}+\frac{1}{2}\right) \pi i\right] \\
& \cdot \sum_{n=0}^{\infty} \frac{\left(3 e^{-(\pi i) / 3} X_{q}^{2 / 3}\right)^{n}}{\Gamma\left(1+\frac{\mu-n}{3}\right) \Gamma(n+1)},
\end{aligned}
$$


and

$$
v_{q, 3}(x, \mu)=\left\{\frac{e^{2(q-1) \mu \pi i}}{3^{\mu} \lambda^{2 \mu}}\right\}^{1 / 3} \sum_{n=0}^{\infty} \frac{\Gamma\left(\frac{n-\mu}{3}\right)\left(3 X_{q}^{2 / 3}\right)^{n}}{3 \Gamma(-\mu) \Gamma(n+1)} .
$$

Their Wronskian, which we shall denote by $w\left(v_{q, j}(x, \mu)\right)$, has the value

$$
W\left(v_{q, j}(x, \mu)\right)=6 \lambda^{2} e^{(1-q) \pi i}\left(\frac{3}{i \lambda}\right)^{2 \mu / 3} .
$$

The solution $v_{q, 3}(x, \mu)$ is of a markedly reduced and simpler form in the special case in which $\mu$ is a non-negative integer. The coefficients in the formula (11.8) are then either indeterminate or vanishing, according as $n$ is, or is not, such as to make $(n-\mu) / 3$ a nonpositive integer. To resolve the indeterminacies the standard identities

$$
\frac{\sin 3 \pi \eta}{\sin \pi \eta}=1+2 \cos 2 \pi \eta
$$

$$
\Gamma(\eta) \Gamma(1-\eta)=\frac{\pi}{\sin \pi \eta}
$$

may be used. The formula (11.8) is therewith found to reduce to

$$
v_{3}(x, \mu)=x^{\mu} \sum_{j=0}^{j \leqq \mu / 3} \frac{\kappa_{j}(\mu)}{\left(\lambda^{2} x^{3}\right)^{j}}, \quad \text { for } \mu=0,1,2, \cdots,
$$

the designation $v_{3}$ having been introduced here to replace $v_{q, 3}$, because the solution is independent of $q$. It is clear that $v_{3}(x, \mu)$ is a polynomial in $x$ and in $1 / \lambda$. The formula (11.11) in this case replaces the relation (11.5) as well as the formula (11.8).

Through the relation (7.10) the forms that have been displayed relate to the differential equation (1.1), as may be summarized in the following terms.

Theorem 5. A differential equation (1.1) admits, for each integer $q$, the set of linearly independent formal solutions $w_{q, j}(z), j=1,2,3$, whose formulas are

$$
\left[\begin{array}{c}
w_{q, j}^{\prime}(z) \\
w_{q, j}^{\prime}(z) \\
w_{q, j}^{\prime \prime}(z) \\
w_{q, j}^{\prime \prime \prime}(z)
\end{array}\right]=M(\nu, \nu)\left[\begin{array}{c}
v_{q, j}(x, \nu) \\
\dot{v}_{q, j}(x, \nu) \\
\ddot{v}_{q, j}(x, \nu)
\end{array}\right],
$$

the functions $v_{q, j}(x, \nu)$ having the formulas (11.7) and (11.8), and being of the asymptotic forms (11.2), (11.3), and (11.5). If $\nu$ is an integer (in which case $\nu=\rho$ ) the formal solution $w_{q, 3}(z)$ is independent of $q$, and, when denoted by $w_{3}(z)$, has the formulas 


$$
\left[\begin{array}{c}
w_{3}(z) \\
w_{3}^{\prime}(z) \\
w_{3}^{\prime \prime}(z) \\
w_{3}^{\prime \prime \prime}(z)
\end{array}\right]=M(\nu, \nu)\left[\begin{array}{l}
v_{3}(x, \nu) \\
\dot{v}_{3}(x, \nu) \\
\ddot{v}_{3}(x, \nu)
\end{array}\right],
$$

the function $v_{3}(x, \nu)$ being given by the formula (11.11).

12. A first integral of the given differential equation. The expressions $w_{q, j}(z, \mu), j=1,2,3$, together with any suitably differentiable function or formal expression $f(z)$, have a Wronskian $\mathscr{W}\left(w_{q, j}(z, \mu), f(z)\right)$ which is representable, in the standard manner, as a four rowed determinant. The determinant which is the Wronskian of the functions $v_{q, j}(x, \mu), j=1,2,3$, is three rowed, but can be supplied with a fourth row and column in which the common element is 1 and the others are 0 . In terms of this representation the relations (7.3)-(7.6) may be seen to insure the equation

$$
W\left(w_{q, j}(z, \mu), f(z)\right)=L(f, \nu, \mu) W\left(v_{q, j}(x, \mu)\right)
$$

in which

$$
L(f, \nu, \mu)=\left|\begin{array}{llll}
A(z, \nu) & \lambda^{-1} B & \lambda^{-2} C & f \\
A^{\prime}+\sigma \mu C & \sigma(A-x C)+\lambda^{-1} B^{\prime} & \lambda^{-1} \sigma B+\lambda^{-2} C^{\prime} & f^{\prime} \\
\lambda H(\nu, \mu) & \lambda J(\nu, \mu) & K(\nu) & f^{\prime \prime} \\
\lambda^{2} \sigma \mu K+\lambda H^{\prime} & -\lambda^{2} x \sigma K+\lambda\left(\sigma H+J^{\prime}\right) & \lambda \sigma J+K^{\prime} & f^{\prime \prime \prime}
\end{array}\right| .
$$

The derivative of the equation (12.1) is

$$
\left.W^{\prime}\left(w_{q, j}(z, \mu), f(z)\right)=L^{\prime}(f, \nu, \mu) w_{q, j}(x, \mu)\right),
$$

because the Wronskian on the right is independent of $z$, as may be inferred either from the form of the differential equation (7.1), or from the evaluation (11.9).

Now the Wronskian on the left of the equation (12.1) is formally differentiable by merely differentiating those of its elements as are third derivatives. And when that has been done, the result is unaffected by the replacement of the consequent elements $w_{q, j}^{\prime \prime \prime}$ and $f^{\prime \prime \prime \prime}$ by the respective expressions $\mathcal{L}\left(w_{q, j}(z, \mu)\right)$ and $\mathscr{L}(f)$. Thus

$$
W^{\prime}\left(w_{q, j}(z, \mu), f(z)\right)=\left|\begin{array}{llll}
w_{q, 1}(z, \mu) & w_{q, 2}(z, \mu) & w_{q, 3}(z, \mu) & f(z) \\
w_{q, 1}^{\prime} & w_{q, 2}^{\prime} & w_{q, 3}^{\prime} & f^{\prime} \\
w_{q, 1}^{\prime \prime} & w_{q, 2}^{\prime \prime} & w_{q, 3}^{\prime \prime} & f^{\prime \prime} \\
\mathscr{L}\left(w_{q, 1}\right) & \mathfrak{L}\left(w_{q, 2}\right) & \mathfrak{L}\left(w_{q, 3}\right) & \mathscr{L}(f)
\end{array}\right| .
$$

This evaluation can be factored, by the use of the relations (7.3)-(7.6), in the manner of the relation (12.1). A comparison of the result with the relation (12.3) shows that 
(12.5) $\quad L^{\prime}(f, \nu, \mu)=\left|\begin{array}{llll}A(z, \nu) & \lambda^{-1} B(z, \nu) & \lambda^{-2} C(z, \nu) & f(z) \\ A^{\prime}+\sigma \mu C & \sigma(A-x C)+\lambda^{-1} B^{\prime} & \lambda^{-1} \sigma B+\lambda^{-2} C^{\prime} & f^{\prime} \\ \lambda H(\nu, \mu) & \lambda J(\nu, \mu) & K(\nu) & f^{\prime \prime} \\ \lambda^{2} S_{1}(\nu, \mu) & \lambda^{2} S_{2}(\nu, \mu) & \lambda S_{3}(\nu, \mu) & \mathfrak{L}(f)\end{array}\right|$.

Consider the relation (12.1), now, with $\nu$ and any formal solution $w(z)$ of the equation (1.1) in the places of $\mu$ and $f(z)$. The Wronskian of $w_{q, j}(z, \nu)$, $j=1,2,3$, and $w(z)$ is constant with respect to $z$, because the equation (1.1) involves no term in $w^{\prime \prime \prime}$. It follows from this, that every formal solution of the equation (1.1) fulfills a relation

$$
L(w, \nu, \nu)=c,
$$

in which $c$ is independent of $z$. The relation (12.6) is thus a "first integral" of the given differential equation.

The expression $L(w, \nu, \nu)$ is a differential form of the third order in $w$. By (12.2) its coefficient of $w^{\prime \prime \prime}$ is the determinant $D_{0}(\nu, \nu)$, whose formula is

$$
D_{0}(\nu, \mu)\left|\begin{array}{lll}
A(z, \nu) & \lambda^{-1} B & \lambda^{-2} C \\
A^{\prime}+\sigma \mu C & \sigma(A-x C)+\lambda^{-1} B^{\prime} & \lambda^{-1} \sigma B+\lambda^{-2} C^{\prime} \\
\lambda H(\nu, \mu) & \lambda J(\nu, \mu) & K(\nu)
\end{array}\right| .
$$

It is verifiable at once that $D_{0}(\nu, \nu)$ is a formal power series in $1 / \lambda$, the $\lambda$-free term of which is

$$
\alpha_{0} \sigma^{3}\left[\left(\alpha_{0}-x \gamma_{0}\right)^{2}+x \beta_{0}^{2}\right] .
$$

That this term is nonvanishing over the $z$-domain is guaranteed by the evaluation (9.11) and the nonvanishing character of $\alpha_{0}(z)$ and $\sigma(z)$. The first integral relation (12.6) is, therefore, nonsingular.

It will be seen directly, from the equation (12.1), that those, and only those, formal solutions $w(z)$ as are linearly dependent upon those of a set $w_{q, j}(z, \nu), j=1,2,3$, fulfill the relation (12.6) with 0 in the place of $c$.

13. Has a complete set of formal solutions been derived? In the total set of functions $v_{q, j}(x, \nu)$, no more than three are linearly independent, since they all solve the differential equation of the third order (7.1). Those with any particular $q$ are three and are linearly independent. It suffices, therefore, to consider only those that are associated with a specific $q$. That fact is projected through the formulas (11.12) to the functions $w_{q, j}(z)$.

The differential equation (1.1) admits a formal solution of the form (3.1) namely one which is a power series in $1 / \lambda$ with coefficients that are analytic in $z$. It is pertinent to inquire whether such a solution may be linearly dependent upon those of a set $w_{q, j}(z), j=1,2,3$. We shall press this inquiry by exploring the consequences of the

Supposition. There is a formal solution w(z) which is a power series in $1 / \lambda$ 
with analytic coefficients, and which is a linear combination of the formal solutions $w_{q, j}(z), j=1,2,3$.

Let $v(x, \nu)$ be defined in terms of the $v_{q, j}(x, \nu)$ by the combination which gives $w(z)$ in terms of the $w_{q, j}(z)$. Then $w(z)$ and $v(x, \nu)$ fulfill the system of equations (7.3)-(7.5) with $\nu$ in the place of $\mu$. The determinant of that system is $D_{0}(\nu, \nu)$, which is obtainable from the formula (12.7). This was seen in $\$ 12$ to be a formal power series in $1 / \lambda$ with analytic coefficients, and with the $\lambda$-free term (12.8), which is different from zero over the $z$-domain. The system is accordingly solvable for $v(x, \nu)$, the solving formula being

$$
v(x, \nu) \frac{1}{D_{0}(\nu, \nu)}\left|\begin{array}{lll}
w(z) & \lambda^{-1} B(z, \nu) & \lambda^{-2} C(z, \nu) \\
w^{\prime} & \sigma(A-x C)+\lambda^{-1} B^{\prime} & \lambda^{-1} \sigma B+\lambda^{-2} C^{\prime} \\
w^{\prime \prime} & \lambda J(\nu, \nu) & K(\nu)
\end{array}\right| .
$$

From this it is to be seen that $v(x, \nu)$ is also a power series in $1 / \lambda$ with analytic coefficients, and, moreover, that the $\lambda$-free terms of $w(z)$ and $v(x, \nu)$ have zeros of the same order at $z=0$. We shall inspect the conformity of this with the differential equation (7.1).

The substitution of a power series in $1 / \lambda$ into the differential equation (7.1) shows the series to be a formal solution if, and only if, it is a multiple (with a multiplier that is independent of $x$ ) of the specific series

$$
\sum_{n=0}^{\infty} \frac{\kappa_{n}(\nu) x^{\nu}}{x^{3 n} \lambda^{2 n}}
$$

in which the values $\kappa_{n}(\nu)$ are those given by the formula (11.6). The coeffcients of this are not analytic if $\nu$ is not an integer. In such a case, therefore, the supposition above is untenable.

If $\nu$ is an integer, the series (13.2) is found to be no more than a polynomial in $1 / \lambda$, in fact precisely the function $v_{3}(x, \nu)$ which is given by the formula (11.11). It thus indeed does have analytic coefficients, its $\lambda$-free term being $x^{\nu}$. The supposition relative to $w(z)$ is therefore in this case endorsed, the $\lambda$-free term of $w(z)$ having at $z=0$ a zero of the order $\nu$. We may draw consequences from this conclusion as follows.

An integral value of $\nu$ implies, through (9.5), that $\nu$ and $\rho$ are the same. The instances to be considered are, therefore, those in which the Theorems 2,3 , or 4 are relevant. If the Theorem 2 is relevant, the formal solution of the form ascribed to $w(z)$ is unique, except for a factor which is independent of $z$, and $w_{*}(z)$ and $w_{3}(z)$ are both such solutions. In this case, therefore,

$$
w_{*}(z) \equiv \Omega(\lambda) w_{3}(z)
$$

with a multiplier $\Omega(\lambda)$ which is independent of $z$.

If the Theorem 3 is relevant, there are formal solutions $w_{*}(z)$ and $w_{\rho, *}(z)$ which are power series in $1 / \lambda$. Of these the former one does not have the form 
inferred for $w(z)$, since it has a $\lambda$-free term which is not zero at $z=0$. This is, therefore, linearly independent of the solutions $w_{q, j}(z)$. That in this case $w_{\rho, *}(z)$ is a multiple of $w_{3}(z)$, can be inferred from the fact that a differential equation (1.1) cannot have five linearly independent formal solutions. The hypotheses of the Theorem 3 are thus incidentally shown to be consistent only with an integral value of $\nu$.

If the Theorem 4 is relevant, the solution of the form (3.1) is effectively unique. Therefore a relation (13.3), with $w_{*}(z)$ as given by the formula (6.8) maintains. That, however, is possible if, and only if, the constant $b_{N}$ is zero, since otherwise the $\lambda$-free terms of $w(z)$ and $w_{*}(z)$ do not have zeros of the same order at $z=0$. We must conclude from this, that when the Theorem 4 is relevant and $\nu$ is an integer, then the constant $b_{N}$ referred to by the theorem is zero. The relation (13.3) then maintains.

We formulate our result as follows.

Theorem 6. A differential equation (1.1) is one for which the formal solutions $w_{*}(z)$ and $w_{q, j}(z), j=1,2,3$, with any $q$ are a complete (fundamental) set, unless it is one for which $\nu$ is an integer and either the Theorem 2 or the Theorem 4 is relevant.

\section{Part III. Differential equations of the distinctive Category}

14. The existence of distinctive equations. The differential equations (1.1) which are exceptional to the Theorem 6 , and for which a complete set of formal solutions has, therefore, not yet been derived, are those for which

(a) $\nu$ is an integer, and

(b) the formal solutions of the type (3.1) with analytic coefficients are all linearly dependent upon one of them, and have initial terms which vanish to the order $\nu$ at the origin.

We shall designate the category of these differential equations (1.1) as the distinctive category. An equation of this category has three linearly independent solutions $w_{q, 1}(z), w_{q, 2}(z)$ and $w_{3}(z)$. A fourth remains, however, to be found.

It is, of course, pertinent to inquire whether, in fact, such equations exist. That they do is shown by the following examples. To the first of these the Theorem 2 is relevant, and to the second one the Theorem 4.

EXAmple 1. Take the differential equation (1.1) for which

$$
\begin{aligned}
& P(z, \lambda)=\left(z+z^{2}\right)(1+2 z)^{2}-\frac{12}{(1+2 z)^{2} \lambda^{2}}, \\
& Q(z, \lambda)=6\left(z+z^{2}\right)(1+2 z)+\frac{72}{(1+2 z)^{3} \lambda^{2}}, \\
& R(z, \lambda)=-4\left(1+3 z+3 z^{2}\right)-\frac{144}{(1+2 z)^{4} \lambda^{2}} .
\end{aligned}
$$


The limiting form (2.3) for this equation is

$$
\left(z+z^{2}\right)(1+2 z)^{2} u^{\prime \prime}+6\left(z+z^{2}\right)(1+2 z) u^{\prime}-4\left(1+3 z+3 z^{2}\right) u=0 .
$$

This has at $z=0$ the exponents 0 and 1 , and respective solutions

$$
u_{0}(z)=\frac{1}{1+2 z}+4\left\{\frac{z+z^{2}}{1+2 z}\right\} \log \left[\frac{z+z^{2}}{(1+2 z)^{2}}\right], \quad u_{1}(z)=\frac{z+z^{2}}{1+2 z} .
$$

Since only one of these solutions is analytic at $z=0$, the differential equation is one for which the Theorem 2 is relevant. The equations (3.4) are found to be fulfilled by $U_{n}(z) \equiv 0$, for $n=1,2,3, \cdots$. Therefore

$$
w_{*}(z)=\frac{z+z^{2}}{1+2 z} .
$$

The formulas (8.2) and (8.3) give, for this differential equation, $x=z+z^{2}$, and $\sigma=1+2 z$. Therewith the equations (7.14) are found to be fulfilled by $\nu=1$, and $A=1 / \sigma, B \equiv C \equiv 0$. The equation (7.1) with $\mu=1$ gives $v_{3}(x, 1) \equiv x$. By the formula (7.3), therefore, $w_{3}(z)=x / \sigma$, and this is identical with $w_{*}(z)$.

EXAmple 2. Take the differential equation (1.1) for which

$$
\begin{aligned}
& P(z, \lambda)=\left(z+\frac{1+z^{2}}{\lambda}+\frac{z}{\lambda^{2}}\right)\left(1+\frac{z}{\lambda}+\frac{1}{\lambda^{2}}\right)^{-1}, \\
& Q(z, \lambda)=\left(\frac{z}{\lambda}+\frac{1}{\lambda^{2}}\right)\left(1+\frac{z}{\lambda}+\frac{1}{\lambda^{2}}\right)^{-1}, \\
& R(z, \lambda)=-\frac{1}{\lambda}\left(1+\frac{z}{\lambda}+\frac{1}{\lambda^{2}}\right)^{-1} .
\end{aligned}
$$

The limiting form (2.3) for this equation is $z u^{\prime \prime}=0$. This has at $z=0$ the exponents 0 and 1 , and the respective solutions $u_{0}(z) \equiv 1, u_{1}=z$. Since these solutions are both analytic, and the relations (6.7) are fulfilled with $a_{1}=1$, $b_{1}=0$, the differential equation is one for which the Theorem 4 is relevant. It is found that $w_{*}(z)=z+1 / \lambda$.

The formulas (8.2) and (8.3) give, in this instance, $x=z$, and $\sigma=1$. Therewith the equations (7.14) are found to be fulfilled by $\nu=1, A \equiv B \equiv 1, C \equiv 0$. The formula $(7.3)$, with $v_{3}(x, 1) \equiv x$, thus shows that $w_{3}(z)$ is identical with $w_{*}(z)$.

15. A perturbation of the given equation. The differential equation (1.1) with which we are now concerned is one with the features (14.1). We propose to consider in place of it the differential equation which is formally solved by the expressions $w(z, \mu)$ given by the formula (7.3) with

$$
\mu=\nu+\frac{\epsilon}{\lambda^{s}},
$$


$\epsilon$ being a parameter which is numerically small and free to approach zero as a limit, and $s$ being a positive integer whose specification we defer. To begin with, we shall determine the form of that differential equation.

By the formulas (7.7) and (7.9), the expressions $H(\nu, \mu)$ and $J(\nu, \mu)$, and therefore $S_{j}(\nu, \mu), j=1,2,3$, are polynomials in $\mu$, all but $S_{1}(\nu, \mu)$ being linear, and that quadratic. Because the equations (7.14) maintain, in virtue of the determinations of $\nu, A, B$ and $C$, it follows that

$$
\begin{aligned}
& S_{1}(\nu, \mu)=\frac{\epsilon}{\lambda^{s+1}} S_{1,1}+\frac{\epsilon^{2}}{\lambda^{2 s}} S_{1,2}, \\
& S_{2}(\nu, \mu)=\frac{\epsilon}{\lambda^{s}} S_{2,1}, \\
& S_{3}(\nu, \mu)=\frac{\epsilon}{\lambda^{s}} S_{3,1},
\end{aligned}
$$

each symbol $S_{j, i}$ signifying a formal power series in $1 / \lambda$ with coefficients that are analytic in $z$.

From the system of equations (7.3), (7.4), (7.5), and (7.8), the functions $v(x, \mu), \dot{v}(x, \mu)$ and $\ddot{v}(x, \mu)$ can be eliminated. The eliminant relation is

$$
L^{\prime}(w(z, \mu), \nu, \mu)=0,
$$

the left-hand member of this having the formula (12.5). The expressions $w(z, \mu)$ thus formally solve the differential equation

$$
D_{0}(\nu, \mu) \mathfrak{L}(w)+D_{1}(\nu, \mu) w^{\prime \prime}+D_{2}(\nu, \mu) w^{\prime}+D_{3}(\nu, \mu) w=0,
$$

namely, on account of (2.7), the equation

$$
w^{\prime \prime \prime \prime}+\lambda^{2}\left\{P(z, \lambda, \mu) w^{\prime \prime}+Q(z, \lambda, \mu) w^{\prime}+R(z, \lambda, \mu) w\right\}=0,
$$

in which

$$
\begin{aligned}
& P(z, \lambda, \mu)=P(z, \lambda)+\frac{D_{1}(\nu, \mu)}{\lambda^{2} D_{0}(\nu, \mu)}, \\
& Q(z, \lambda, \mu)=Q(z, \lambda)+\frac{D_{2}(\nu, \mu)}{\lambda^{2} D_{0}(\nu, \mu)}, \\
& R(z, \lambda, \mu)=R(z, \lambda)+\frac{D_{3}(\nu, \mu)}{\lambda^{2} D_{0}(\nu, \mu)} .
\end{aligned}
$$

$D_{i}(\nu, \mu)$ is, of course, the cofactor of the element in the $(4-i)$ th row and fourth column of the determinant in (12.5).

Because of the evaluations (15.2), and the fact that $H(\nu, \mu)$ and $J(\nu, \mu)$ are polynomials in $\epsilon$, it is found that 


$$
\begin{aligned}
& D_{0}(\nu, \mu)=D_{0}(\nu, \nu)+\frac{\epsilon}{\lambda^{s+1}} D_{0,1}+\frac{\epsilon^{2}}{\lambda^{2 s+2}} D_{0,2}, \\
& D_{1}(\nu, \mu)=\frac{\epsilon}{\lambda^{s-1}} D_{1,1}+\frac{\epsilon^{2}}{\lambda^{2 s}} D_{1,2}, \\
& D_{2}(\nu, \mu)=\frac{\epsilon}{\lambda^{s-2}} D_{2,1}+\frac{\epsilon^{2}}{\lambda^{2 s-1}} D_{2,2}+\frac{\epsilon^{3}}{\lambda^{3 s}} D_{2,3}, \\
& D_{3}(\nu, \mu)=\frac{\epsilon}{\lambda^{s-2}} D_{3,1}+\frac{\epsilon^{2}}{\lambda^{2 s-2}} D_{3,2}+\frac{\epsilon^{3}}{\lambda^{3 s-1}} D_{3,3},
\end{aligned}
$$

each symbol $D_{i, j}$ signifying a formal power series in $1 / \lambda$ with coefficients that are analytic in $z$. Moreover, the $\lambda$-free term of $D_{0}(\nu, \mu)$ is the function (12.8), which does not vanish anywhere in the $z$-domain. The formulas (15.5) thus have, more explicitly, the structures

$$
\begin{aligned}
& P(z, \lambda, \mu)=P(z, \lambda)+\frac{\epsilon}{\lambda^{s}} p(z, \lambda)+\frac{\epsilon^{2}}{\lambda^{2 s}} \mathcal{Q}(z, \lambda, \epsilon), \\
& Q(z, \lambda, \mu)=Q(z, \lambda)+\frac{\epsilon}{\lambda^{s}} q(z, \lambda)+\frac{\epsilon^{2}}{\lambda^{2 s}} Q(z, \lambda, \epsilon), \\
& R(z, \lambda, \mu)=R(z, \lambda)+\frac{\epsilon}{\lambda^{s}} r(z, \lambda)+\frac{\epsilon^{2}}{\lambda^{2 s}} \mathcal{R}(z, \lambda, \epsilon),
\end{aligned}
$$

in which

$$
\begin{aligned}
& p(z, \lambda)=\sum_{n=0}^{\infty} \frac{p_{n}(z)}{\lambda^{n}}, \\
& q(z, \lambda)=\sum_{n=0}^{\infty} \frac{q_{n}(z)}{\lambda^{n}}, \\
& r(z, \lambda)=\sum_{n=0}^{\infty} \frac{r_{n}(z)}{\lambda^{n}},
\end{aligned}
$$

the functions $p_{n}(z), q_{n}(z)$ and $r_{n}(z)$ being analytic, and $\mathcal{P}, Q$ and $Q$ being formal power series in $1 / \lambda$ whose coefficients are analytic in $z$ and in $\epsilon$.

The differential equation (15.4) manifestly reduces to the given equation (1.1) when $\epsilon \rightarrow 0$, namely when $\mu \rightarrow \nu$. It is of the same type as (1.1), and therefore has a solution $w_{*}(z, \mu)$ which is of the type (3.1) and reduces to the solution $w_{*}(z)$ of the equation (1.1) when $\mu \rightarrow \nu$. By construction it has the formal solutions $w_{q, j}(z, \mu), j=1,2,3$, which reduce to the solutions $w_{q, j}(z)$ of the equation (1.1) when $\mu \rightarrow \nu$. It is not of the distinctive category when $\epsilon \neq 0$, for in the formula (7.3) which solves it $\mu$ has the nonintegral value (15.1). For it, therefore, the expressions $w_{*}(z, \mu)$ and $w_{q, j}(z, \mu), j=1,2,3$, are a com- 
plete set of formal solutions. We shall call the equation (15.4) the perturbed equation.

16. A solution $w_{q, 4}(z)$ of the given differential equation. For a differential equation (1.1) of the distinctive category, such as we are presently considering, a relation (13.3) maintains, and may be taken to define the multiplier $\Omega(\lambda)$. That this multiplier is a formal power series in $1 / \lambda$, follows from the fact that it is independent of $z$, whereas at a point $z$ which is near but not at the origin both $w_{3}(z)$ and $w_{*}(z)$ are such series with $\lambda$-free terms which are different from zero.

Consider the perturbed equation, namely the Equation (15.4) with $\mu \neq \nu$. It has solutions $w_{*}(z, \mu)$ and $w_{q .3}(z, \mu)$, and therefore also the solution

$$
\lambda^{1-s}\left[\frac{w_{*}(z, \mu)-\Omega(\lambda) w_{q, 3}(z, \mu)}{\mu-\nu}\right] .
$$

The limiting form of this as $\mu \rightarrow \nu$ is a solution of the equation (1.1), and evidently has the form

$$
\lambda^{1-8}\left[\left.\frac{\partial w_{*}(z, \mu)}{\partial \mu}\right|_{\mu=\nu}-\left.\Omega(\lambda) \frac{\partial w_{q, 3}(z, \mu)}{\partial \mu}\right|_{\mu=\nu}\right] .
$$

The relation (15.4) with $\lambda^{1-s} w_{*}(z, \mu)$ in the place of $w$, is a formal identity in $z, \lambda$ and $\mu$. Its derivative with respect to $\mu$ has, at $\mu=\nu$, the form

$\mathcal{L}\left(\left.\lambda^{1-s} \frac{\partial w_{*}(z, \mu)}{\partial \mu}\right|_{\mu=\nu}\right)+\lambda^{3-s}\left\{p(z, \lambda) w_{*}^{\prime \prime}(z)+q(z, \lambda) w_{*}^{\prime}(z)+r(z, \lambda) w_{*}(z)\right\}=0$,

in which the coefficients are those of the formulas (15.7). Thus

$$
\lambda^{1-s} \partial w_{*}(z, \mu) /\left.\partial \mu\right|_{\mu=\nu}
$$

is a particular solution of the nonhomogeneous differential equation

$$
\mathscr{L}(V)=-\lambda^{3-x}\left\{p(z, \lambda) w_{*}^{\prime \prime}(z)+q(z, \lambda) w_{*}^{\prime}(z)+r(z, \lambda) w_{*}(z)\right\} .
$$

Let $V_{*}(z)$ be any formal solution of this differential equation. The difference

$$
\left[V_{*}(z)-\left.\lambda^{1-s} \frac{\partial w_{*}(z, \mu)}{\partial \mu}\right|_{\mu=\nu}\right]
$$

is then a formal solution of the "reduced" homogeneous counterpart, namely of the equation (1.1), and since the expression (16.1) is also such a solution the sum of these two expressions, which we shall denote by $w_{q, 4}(z)$, is similarly so. The formula

$$
w_{q, 4}(z)=V_{*}(z)-\left.\lambda^{1-s} \Omega(\lambda) \frac{\partial w_{q, 3}(z, \mu)}{\partial \mu}\right|_{\mu=\nu}
$$


thus yields a solution of the given differential equation (1.1).

Our reason for preferring the solution (16.4) over the more immediately derived one (16.1), lodges in the fact that the term $\partial w_{*}(z, \mu) /\left.\partial \mu\right|_{\mu_{-\nu}}$ of the latter depends upon a determination of $w_{*}(z, \mu)$ as that particular formal solution of the equation (15.4) which reduces, when $\mu \rightarrow \nu$, to the particular $w_{*}(z)$ which enters, through (13.3), into the determination of $\Omega(\lambda)$. The expression $V_{*}(z)$, by contrast, is not subject to such a boundary condition, but may be taken as any convenient formal solution of the differential equation (16.2).

17. A determination of $V_{*}(z)$. The expression $V_{*}(z)$ has been prescribed to be a formal solution of the differential equation (16.2). Let it be taken in the form

$$
V_{*}(z)=\sum_{n=0}^{\infty} \frac{V_{n}(z)}{\lambda^{n}} .
$$

By this, along with (3.1), the equation (16.2) is formally fulfilled if for each $n$

$$
P_{0} V_{n}^{\prime \prime}+Q_{0} V_{n}^{\prime}+R_{0} V_{n}=-h_{n}(V)-\mathfrak{h}_{n}(U),
$$

with $h_{n}(V)$ as given by the formula (3.3), and with

$$
\begin{array}{ll}
\mathfrak{h}_{n}(U) \equiv 0, & \text { for } n<s-1, \\
\mathfrak{h}_{n}(U)=\sum_{j=0}^{n+1-s}\left\{p_{j} U_{n+1-s-j}^{\prime \prime}+q_{j} U_{n+1-s-j}^{\prime}+r_{j} U_{n+1-s-j}\right\}, & \text { for } n \geqq s-1 .
\end{array}
$$

In the manner of $\S 3$, it follows that with

$$
\mathfrak{H}_{n}(U, u)=\int \frac{u \theta \mathfrak{h}_{n}(U)}{-P_{0}} d z
$$

the solving formula is

$$
V_{n}(z)=u(z) \int \frac{H_{n}(V, u)+\mathfrak{K}_{n}(U, u)}{u^{2} \theta} d z+c_{n}^{*} u_{0}(z)+k_{n}^{*} u_{\rho}(z),
$$

$c_{n}^{*}$ and $k_{n}^{*}$ being constants of integration. We shall show that with these constants suitably disposed the functions $V_{n}(z)$ are analytic.

To a differential equation of the distinctive category either the Theorem 2 or the Theorem 4 is relevant. We must consider these cases separately, and shall take them in turn. For a differential equation (1.1) to which the Theorem 2 is relevant, let the relation (15.1) be adopted with $s=1$. With the use of the formula (2.5), and with $u(z) \equiv u_{\rho}(z)$ and $k_{n}^{*}=0$, the formula (17.5) assumes the form

$$
V_{n}(z)=u_{\rho}(z) \int \frac{H_{n}\left(V, u_{\rho}\right)+\mathfrak{C}_{n}\left(U, u_{\rho}\right)+c_{n}^{*}}{u_{\rho}^{2} \theta} d z .
$$


On the basis of this it can be shown, in the manner of $\$ 5$, that with a suitable choice of $c_{n}^{*}$ the function $V_{n}(z)$ is analytic.

A differential equation (1.1) to which the Theorem 4 is relevant has ipso facto a positive integer $N$ associated with it by the relations (6.7), and when it is of the distinctive category, then $b_{N}=0$. Let the relation (15.1) be adopted, in this case, with $s=N+1$. We shall consider the relations

$$
V_{j}(z)=\Delta_{j}(z)+\sum_{i=0}^{j}\left\{c_{i}^{*} \Phi_{j-i}(z)+k_{i}^{*} \Psi_{j-i}(z)\right\},
$$

in which $\Phi_{n}(z)$ and $\Psi_{n}(z)$ have the formulas (6.1), and

$$
\begin{aligned}
& \Delta_{0}(z) \equiv 0, \\
& \Delta_{j}(z)=u_{0}(z) \int \frac{H_{j}\left(\Delta, u_{0}\right)+\mathfrak{K}_{j}\left(U, u_{0}\right)-a_{j}^{*} \log z}{u_{0}^{2} \theta}, j=1,2,3, \cdots,
\end{aligned}
$$

the constants $a_{j}^{*}$ being those for which the functions (17.8) are analytic. That such constants exist is demonstrable in the manner of $\S 6$. It is readily seen that $\Delta_{j}(z) \equiv 0$, and $a_{j}^{*}=0$, for $j<N$.

The relation (17.7) is valid when $j=0$. Suppose it to be so when $j<n$. Then, by the lemma of $\S 4$,

$$
H_{n}\left(V, u_{0}\right)=H_{n}\left(\Delta, u_{0}\right)+\sum_{i=0}^{n-1}\left\{c_{i}^{*} H_{n-i}\left(\Phi, u_{0}\right)+k_{i}^{*} H_{n-i}\left(\Psi, u_{0}\right)\right\},
$$

whereas, in virtue of this and the relations (6.1) and (17.8), the formula (17.5) assumes the form

$$
\begin{aligned}
V_{n}(z)= & \Delta_{n}(z)+\sum_{i=0}^{n}\left\{c_{i}^{*} \Phi_{n-i}(z)+k_{i}^{*} \Psi_{n-i}(z)\right\} \\
& +\left\{a_{n}{ }^{*}+\sum_{i=0}^{n-1}\left[c_{i}^{*} a_{n-i}+k_{i}^{*} b_{n-i}\right]\right\} u_{0}(z) \int \frac{\log z}{u_{0}^{2} \theta} d z .
\end{aligned}
$$

This reduces to the relation (17.7) with $j=n$, and thus establishes these relations for all $j$, if the constants $c_{i}^{*}$ and $k_{i}^{*}$ are assigned values which fulfill the equations

$$
a_{n}^{*}+\sum_{i=0}^{n-1}\left[c_{i}^{*} a_{n-i}+k_{i}^{*} b_{n-i}\right]=0, \quad n=1,2,3, \cdots .
$$

Because $a_{j}=b_{j}=a_{j}^{*}=0$ for $j<N$, this system of equations (17.9), whose solution values $c_{j}^{*}, k_{j}^{*}$ yield analytic determinations of the functions $V_{n}(z)$, can be alternatively written

$$
\sum_{j=. N}^{n}\left\{a_{j} c_{n-j}^{*}+b_{j} k_{n-j}^{*}\right\}=-a_{n}^{*}, \quad n=N, N+1, N+2, \cdots .
$$


18. The form of the solution $w_{q, 4}(z)$. The formula (16.4) involves the $\mu$-derivative of the expression $w_{q, 3}(z, \mu)$, whereas the formulas for this latter are found from (7.10) to be

$$
\left[\begin{array}{l}
\frac{\partial}{\partial \mu} w_{q, 3} \\
\frac{\partial}{\partial \mu} w_{q, 3}^{\prime} \\
\frac{\partial}{\partial \mu} w_{q, 3}^{\prime \prime} \\
\frac{\partial}{\partial \mu} w_{q, 3}^{\prime \prime \prime}
\end{array}\right]=M(\nu, \mu)\left[\begin{array}{l}
\frac{\partial}{\partial \mu} v_{q, 3} \\
\frac{\partial}{\partial \mu} \dot{v}_{q, 3} \\
\frac{\partial}{\partial \mu} \ddot{v}_{q, 3}
\end{array}\right]+M_{2}(\nu, \mu)\left[\begin{array}{l}
v_{q, 3} \\
\dot{v}_{q, 3} \\
\ddot{v}_{q, 3}
\end{array}\right]
$$

with

$$
M_{2}(\nu, \mu)=\frac{\partial}{\partial \mu} M(\nu, \mu)
$$

We therefore turn our attention to the function $v_{q, 3}(x, \mu)$, writing its formula (11.8) so as to display its dependency upon $\mu$ thus,

$$
v_{q, 3}(x, \mu)=G_{q}^{\mu} \sum_{n=0}^{\infty} g_{n}(\mu) \frac{\left(3 X_{q}^{2 / 3}\right)^{n}}{3 \Gamma(n+1)},
$$

with

$$
G_{q}=3^{-1 / 3} \lambda^{-2 / 3} e^{2(q-1) \times i / 3}
$$

and

$$
g_{n}(\mu)=\Gamma\left(\frac{n-\mu}{3}\right) / \Gamma(-\mu) .
$$

The differentiation with respect to $\mu$, coupled with the fact that $v_{q, 3}(x, \mu)$ reduces to $v_{3}(x, \nu)$ when $\mu$ assumes the integral value $\nu$, yields the evaluation

$$
\left.\frac{\partial}{\partial \mu} v_{q, 3}(x, \mu)\right|_{\mu=\nu}=v_{3}(x, \nu) \log G_{q}+y_{q}(x),
$$

with

$$
y_{q}(x)=G_{q}^{\nu} \sum_{n=0}^{\infty} g_{n}^{\prime}(\nu) \frac{\left(3 X^{2 / 3}\right)^{n}}{3 \Gamma(n+1)} .
$$

The formula (18.5) is disadvantageous for the evaluation of $g_{n}^{\prime}(\nu)$, be- 
cause it gives an indeterminate result. It may, however, be alternatively written as

$$
g_{n}(\mu)\left\{\begin{array}{l}
=-\Gamma(1+\mu) \Gamma\left(\frac{n-\mu}{3}\right) \frac{\sin \mu \pi}{\pi}, \\
=\frac{(-1)^{n} \Gamma(1+\mu)}{\Gamma(1+(\mu-n) / 3)}\left\{1+2 \cos 2 \pi\left(\frac{\mu-n}{3}\right)\right\},
\end{array}\right.
$$

by virtue of the identities (11.10). From these formulas it is found that

(18.8) $g_{n}^{\prime}(\nu)$

$$
\left\{\begin{array}{l}
=(-1)^{2+1} \Gamma(1+\nu) \Gamma\left(\frac{n-\nu}{3}\right), \\
\text { when } \frac{n-\nu}{3} \neq 0,-1,-2, \cdots, \\
=3(-1)^{n} \frac{d}{d \nu}\left\{\frac{\Gamma(1+\nu)}{\Gamma(1+(\nu-n) / 3)}\right\},
\end{array}\right.
$$

$$
\text { when } \frac{n-\nu}{3}=0,-1,-2, \cdots .
$$

For values of $\arg \left(\lambda x^{3 / 2}\right)$ to which the relation (11.5) pertains, it is to be seen that

$$
\begin{aligned}
& \left.\frac{\partial}{\partial \mu} v_{1,3}(x, \mu)\right|_{\mu=\nu} \sim v_{3}(x, \nu) \log x+\eta_{0}(x), \\
& \left.\frac{\partial}{\partial \mu} \dot{i}_{q, 3}(x, \mu)\right|_{\mu=\nu} \sim \dot{i}_{3}(x, \nu) \log x+\eta_{1}(x), \\
& \left.\frac{\partial}{\partial \mu} \ddot{i}_{q, 3}(x, \mu)\right|_{\mu=\nu} \sim \ddot{i}_{3}(x, \nu) \log x+\eta_{2}(x),
\end{aligned}
$$

with

$$
\begin{aligned}
& \eta_{11}(x)=x^{\nu} \sum_{n=0}^{\infty} \frac{\kappa_{n}^{\prime}(\nu)}{\left(\lambda^{2} x^{3}\right)^{n}}, \\
& \eta_{1}(x)=x^{\nu-1} \sum_{n=0}^{\infty} \frac{\kappa_{n}(\nu)+(\nu-3 n) \kappa_{n}^{\prime}(\nu)}{\left(\lambda^{2} x^{3}\right)^{n}}, \\
& \eta_{2}(x)=x^{\nu-2} \sum_{n=0}^{\infty} \frac{(2 \nu-6 n-1) \kappa_{n}(\nu)+(\nu-3 n)(\nu-3 n-1) \kappa_{n}^{\prime}(\nu)}{\left(\lambda^{2} x^{3}\right)^{n}} .
\end{aligned}
$$

The formula (11.6) is suitable for the calculation of $\kappa_{n}^{\prime}(\nu)$ when $3 n \leqq \nu$, but is otherwise advantagcously replaced by the alternative 


$$
\kappa_{n}(\mu)=(-1)^{n+1} \frac{\Gamma(1+\mu) \Gamma(3 n-\mu) \sin \mu \pi}{3^{n} \pi \Gamma(n+1)} .
$$

It is thus found that

$$
\kappa_{n}^{\prime}(\nu) \begin{cases}=\frac{1}{3^{n} \Gamma(n+1)} \frac{d}{d \nu}\left\{\frac{\Gamma(1+\nu)}{\Gamma(1+\nu-3 n)}\right\}, & \text { when } 3 n \leqq \nu, \\ =(-1)^{\nu+n-1} 3^{-n} \Gamma(1+\nu) \Gamma(3 n-\nu), & \text { when } 3 n>\nu .\end{cases}
$$

The right-hand member of the relation (18.1) at $\mu=\nu$ is shown by the formulas (18.6) and (18.9) to have the form

$$
\left[M(\nu, \nu) \log G_{q}+M_{2}(\nu, \nu)\right]\left[\begin{array}{l}
v_{3}(x, \nu) \\
\dot{v}_{3}(x, \nu) \\
\ddot{v}_{3}(x, \nu)
\end{array}\right]+M(\nu, \nu)\left[\begin{array}{l}
y_{q}(x) \\
\dot{y}_{q}(x) \\
\ddot{y}_{q}(x)
\end{array}\right]
$$

and to have the asymptotic form that is obtainable therefrom by replacing $G_{q}, y(x), \dot{y}(x)$ and $\ddot{y}(x)$ respectively by $x, \eta_{0}(x), \eta_{1}(x)$ and $\eta_{2}(x)$. We may formulate the conclusion to be drawn from these results together with the formula (16.4) thus:

Theorem 7. A differential equation (1.1) of the distinctive category admits. for each integer $q$, a formal solution $w_{q, 4}(\boldsymbol{z})$ whose formulas are

$$
\begin{gathered}
{\left[\begin{array}{c}
w_{q, 4}(z) \\
w_{q, 4}^{\prime}(z) \\
w_{q, 4}^{\prime \prime}(z) \\
w_{q, 4}^{\prime \prime \prime}(z)
\end{array}\right]=\left[\begin{array}{l}
V_{*}(z) \\
V_{*}^{\prime}(z) \\
V_{*}^{\prime \prime}(z) \\
V_{*}^{\prime \prime \prime}(z)
\end{array}\right]-\lambda^{1-s} \Omega(\lambda)\left[M(\nu, \nu) \log G_{q}+M_{2}(\nu, \nu)\right]\left[\begin{array}{l}
v_{3}(x, \nu) \\
\dot{v}_{3}(x, \nu) \\
\ddot{v}_{3}(x, \nu)
\end{array}\right]} \\
-\lambda^{1-s} \Omega(\lambda) M(\nu, \nu)\left[\begin{array}{l}
y_{q}(x) \\
\dot{y}_{q}(x) \\
\ddot{y}_{q}(x)
\end{array}\right] .
\end{gathered}
$$

In the domain

$$
(q-2) \pi+\epsilon_{0} \leqq \arg \left(\lambda x^{3 / 2}\right) \leqq q \pi-\epsilon_{0},
$$

the asymptotic formulas obtainable from (18.12) by writing $x, \eta_{0}(x), \eta_{1}(x)$ and $\eta_{2}(x)$ in the places of $G_{q}, y(x), \dot{y}(x)$, and $\ddot{y}(x)$ respectively maintain. The equation also has the formal solutions $w_{q, 1}(z), w_{q, 2}(z)$ and $w_{3}(z)$ described by the Theorem 5 .

Part IV. The Construction of a Related Equation

19. Truncations of the formal solutions. The formal solutions that have been derived for the differential equation (1.1) all involve power series in $1 / \lambda$ which are in general divergent. They are, therefore, not functions, and hence not actual solutions. Functions are obtainable from them by the trun- 
cation of their infinite series, but these do not fulfill the differential equation. We shall show, however, that if the truncations are appropriately made the resulting functions do fulfill a differential equation which is of the same type as (1.1), and which has coefficients that coincide with those of the equation (1.1) to the extent of all terms to prescribed degrees in $1 / \lambda$. This differential equation-which we shall call the related equation-is solvable, since it is, in fact, derived from explicitly known solutions.

The construction of a related equation has been found, for whole classes of linear differential equations, to open the way to a determination of the asymptotic solution forms. Analytic method is available for showing that the resemblance between the two differential equations insures a corresponding resemblance in appropriately associated solutions $\left({ }^{5}\right)$.

Let $m$ be chosen as a positive integer which exceeds a value later to be specified. Except for this condition, the choice may be made at pleasure; in particular $m$ may be taken arbitrarily large. The effect of replacing the elements $U_{n}(z), \nu_{n}, \alpha_{n}(z), \beta_{n}(z)$ and $\gamma_{n}(z)$ for which $n>m$ by zeros, is to truncate the infinite series in the formulas (3.1), (7.12) and (7.13) at their terms in $(1 / \lambda)^{m}$. We shall make these truncations, and shall distinguish the functions which are thereby obtained through the use of the superscribed accent $\widehat{ }$, thus

$$
\begin{aligned}
\hat{w}_{*}(z) & =\sum_{n=0}^{m} \frac{U_{n}(z)}{\lambda^{n}}, \\
\hat{v}(z) & =\sum_{n=0}^{m} \frac{\nu_{n}}{\lambda^{n}}
\end{aligned}
$$

and

$$
\begin{aligned}
& \hat{A}(z, \nu)=\sum_{n=0}^{m} \frac{\alpha_{n}(z)}{\lambda^{n}}, \\
& \hat{B}(z, \nu)=\sum_{n=0}^{m} \frac{\beta_{n}(z)}{\lambda^{n}}, \\
& \hat{C}(z, \nu)=\sum_{n=0}^{m} \frac{\gamma_{n}(z)}{\lambda^{n}} .
\end{aligned}
$$

The functions $\hat{w}_{q, j}(z, \mu), j=1,2,3, \hat{H}(\nu, \mu), \hat{J}(\nu, \mu), \hat{K}(\nu), \hat{S}_{j}(\nu, \mu), j=1,2,3$, the matrix $\hat{M}(\nu, \mu)$, and the determinant $\hat{L}(f, \nu, \mu)$, shall be those that are obtainable from the respective formulas (7.3), (7.7), (7.9), (7.11) and (12.2) by the replacements of $A, B$, and $C$ by $\hat{A}, \hat{B}$ and $\hat{C}$. It is found that

(5) The paper [1] includes this analysis for differential equations (1.1) except those of the distinctive category. 


$$
\left[\begin{array}{c}
\hat{w}_{q, j}(z, \mu) \\
\hat{w}_{q, j}^{\prime}(z, \mu) \\
\hat{w}_{q, j}^{\prime \prime}(z, \mu) \\
\hat{w}_{q, j}^{\prime \prime \prime}(z, \mu)
\end{array}\right]=\hat{M}(\nu, \mu)\left[\begin{array}{c}
v_{q, j}(x, \mu) \\
\dot{v}_{q, j}(x, \mu) \\
\ddot{v}_{q, j}(x, \mu)
\end{array}\right]
$$

and

(19.5) $\mathcal{L}\left(\hat{w}_{q, j}(z)\right)=\lambda^{2} \hat{S}_{1}(\nu, \hat{\nu}) v_{q, j}(x, \hat{\nu})+\lambda^{2} \hat{S}_{2}(\nu, \hat{\nu}) \dot{v}_{q, j}(x, \hat{\nu})+\lambda \hat{S}_{3}(\nu, \hat{\nu}) \ddot{v}_{q, j}(x, \hat{\nu})$.

Also, it may be drawn from the relations (12.1) and (12.3) that

$$
\begin{aligned}
W\left(\hat{w}_{q, j}(z, \mu), f(z)\right) & =\hat{L}(f, \nu, \mu) W\left(v_{q, j}(x, \mu)\right), \\
W^{\prime}\left(\hat{w}_{q, j}(z, \mu), f(z)\right) & =\hat{L}^{\prime}(f, \nu, \mu) W\left(v_{q, j}(x, \mu)\right) .
\end{aligned}
$$

The function $\lambda^{-2} \hat{L}\left(\hat{w}_{*}, \nu, \hat{\nu}\right)$, which is thus obtainable from the formula (12.2), is a polynomial in $1 / \lambda$ which coincides to the extent of its terms to the $m$ th degree in $1 / \lambda$ with the terms of the formal power series $\lambda^{-2} L\left(w_{*}, \nu, \nu\right)$. This latter, by (12.6), is independent of $z$. It follows that the first $(m+1)$ coefficients of the polynomial $\lambda^{-2} \hat{L}\left(\hat{w}_{*}, \nu, \hat{\nu}\right)$ are constants.

20. The related equation in the general case. When the formal solution $w_{*}(z)$ is linearly independent of $w_{q, 3}(z)$ it fulfills the first integral relation (12.6) with $c$ different from zero. There is accordingly an integer $m_{0}$ such that

$$
\lambda^{-2} L\left(w_{*}, \nu, \nu\right)=\sum_{n=m_{0}}^{\infty} \frac{c_{n}}{\lambda^{n}}, \quad \text { with } c_{m_{0}} \neq 0,
$$

and $m_{0}$ is hereby determined. We shall impose upon the choice of the integer $m$ the condition that $m>m_{0}$. It follows then that

$$
\lambda^{-2} \hat{L}\left(\hat{w}_{*}, \nu, \hat{\nu}\right)=\sum_{n=m_{0}}^{m} \frac{c_{n}}{\lambda^{n}}+\frac{E(z, \lambda)}{\lambda^{m+1}},
$$

with $E(z, \lambda)$ denoting a function that is bounded over the $z$-domain when $|\lambda|$ is sufficiently large $\left({ }^{6}\right)$. It follows that

$$
\frac{\hat{L}^{\prime}\left(\hat{w}_{*}, \nu, \hat{\nu}\right)}{\hat{L}\left(\hat{w}_{*}, \nu, \hat{v}\right)}=\frac{E(z, \lambda)}{\lambda^{m-m_{0}+1}} .
$$

Consider the relation

$$
W^{\prime}\left(\hat{w}_{q, j}(z), w\right)-\frac{W^{\prime}\left(\hat{w}_{q, j}(z), \hat{w}_{*}(z)\right)}{W\left(\hat{w}_{q, j}(z), \hat{w}_{*}(z)\right)} W\left(\hat{w}_{q, j}(z), w\right)=0 .
$$

It is a linear differential equation of the fourth order, and is seen at once to be fulfilled when $w$ is taken to be any one of the functions $w_{*}(z)$, or $\hat{v}_{q, j}(z)$.

$\left.{ }^{(}\right)$We shall henceforth use the symbol $E(z, \lambda)$ as a generic designation for functions that are so bounded. 
It is, therefore, the differential equation of which the functions $\hat{w}_{*}(z)$ and $\hat{w}_{q, j}(z), j=1,2,3$, are a complete set of solutions. We shall show that it is of the type (1.1) and that it has coefficients which coincide with those of the equation (1.1) to the terms of the $\left(m-m_{0}\right)$ th degree in $1 / \lambda$.

By use of the relations (19.6) the equation (20.4) is immediately reducible to the form

$$
\hat{L}^{\prime}(w, \nu, \hat{\nu})-\frac{\hat{L}^{\prime}\left(\hat{w}_{*}, \nu, \hat{\nu}\right)}{\hat{L}\left(\hat{w}_{*}, \nu, \hat{\nu}\right)} L(w, \nu, \hat{\nu})=0 .
$$

Its independence of $q$ is hereby clearly shown. The function $\hat{S}_{j}(\nu, \hat{\nu})$ and the respective formal expression $S_{j}(\nu, \nu)$ coincide to their terms in $(1 / \lambda)^{m}$, and the latter vanishes, as is shown by (7.14). Thus

$$
\hat{S}_{j}(\nu, \hat{\nu})=\frac{E(z, \lambda)}{\lambda^{m+1}}, \quad j=1,2,3,
$$

whereas by virtue of this it may be drawn from the truncated formula (12.5) that

$$
\hat{L}^{\prime}(w, \nu, \hat{\nu})=\hat{D}_{0}(\nu, \hat{\nu})\left\{\mathcal{L}(w)+\lambda^{-m-1}\left[\lambda E w^{\prime \prime}+\lambda^{2} E w^{\prime}+\lambda^{2} E w\right]\right\} .
$$

The truncated formula (12.2) yields

$$
\hat{L}(w, \nu, \hat{\nu})=\hat{D}_{0}(\nu, \hat{\nu})\left\{w^{\prime \prime \prime}+\lambda E w^{\prime \prime}+\lambda^{2} E w^{\prime}+\lambda^{2} E w\right\} .
$$

The related equation (20.5), after division by $\hat{D}_{0}(\nu, \hat{\nu})$, is shown by the evaluations (20.7), (20.3), and (20.8), to have the form

$$
\mathfrak{L}(w)+\frac{1}{\lambda^{m-m_{0}+1}}\left\{E w^{\prime \prime \prime}+\lambda E w^{\prime \prime}+\lambda^{2} E w^{\prime}+\lambda^{2} E w\right\}=0 .
$$

That its coefficients coincide with those of the given differential equation (1.1) to terms of the order of $(1 / \lambda)^{m-m_{0}}$ has thus been established.

21. A related equation in the case of the distinctive category. For a differential equation (1.1) for which $\nu$ is an integer and the formal solutions $w_{*}(z)$ and $w_{3}(z)$ are linearly dependent, the formal solution $w_{q, 4}(z)$ with the formula (16.4) has been derived. This fulfills the first integral relation (12.6), namely

$$
L\left(w_{q, 4}, \nu, \nu\right)=c
$$

with a right-hand member $c$ that is independent of $z$ and is not 0 . We are interested in the form of $c$ as it depends upon $\lambda$.

By virtue of the formula (16.4), it is seen directly that

$$
L\left(w_{q, 4}, \nu, \nu\right)=L\left(V_{*}, \nu, \nu\right)-\lambda^{1-s} \Omega(\lambda) L\left(\left[\frac{\partial}{\partial \mu} w_{4,3}(z, \mu)\right]_{\mu=\nu}, \nu, \nu\right) .
$$


From the equation (12.1) it can be inferred that the relation

$$
L\left(w_{q, 3}(z, \mu), \nu, \mu\right)=0,
$$

is an identity in $\mu$. The derivative of this identity yields the fact that

$$
L\left(\left[\frac{\partial}{\partial \mu} w_{q, 3}(z, \mu)\right]_{\mu=\nu}, \nu, \nu\right)+\left[\frac{\partial}{\partial \mu} L\left(w_{3}(z), \nu, \mu\right)\right]_{\mu=\nu}=0,
$$

whereas hereby the evaluation (21.2) is made replaceable by

$$
L\left(w_{q, 4}, \nu, \nu\right)=L\left(V_{*}, \nu, \nu\right)+\lambda^{1-s} \Omega(\lambda)\left[\frac{\partial}{\partial \mu} L\left(w_{3}(z), \nu, \mu\right)\right]_{\mu=\nu} .
$$

Now $w_{3}(z)$ is a formal power series in $1 / \lambda$, and by consequence of that $\lambda^{-2} L\left(w_{3}(z), \nu, \mu\right)$ is also such a series, the coefficients of which are polynomials in $\mu$. The element $\lambda^{-2}\left[(\partial / \partial \mu) L\left(w_{3}(z), \nu, \mu\right)\right]_{\mu=\nu}$, as well as $\lambda^{-2} L\left(V_{*}, \nu, \nu\right)$ and $\lambda^{1-s} \Omega(\lambda)$, is thus a $1 / \lambda$ power series. By (21.4), therefore, a more explicit form of the equation (21.1) is

$$
\lambda^{-2} L\left(w_{q, 4}, \nu, \nu\right)=\sum_{n=m_{0}}^{\infty} \frac{c_{n}}{\lambda^{n}}, \quad \text { with } c_{m_{0}} \neq 0,
$$

and by this the integer $m_{0}$ is defined.

For each differential equation (1.1) of the category now in question an integer $s$ was defined in $\$ 17$. The condition we shall impose upon the truncations signified by the formulas (19.1)-(19.3) shall be that $m$ exceed both $m_{0}$ and $s$. With such an $m$ we shall designate by $\hat{V}_{*}(z)$ and $\hat{\Omega}(\lambda)$ the truncations of the formal power series $V_{*}(z)$ and $\Omega(\lambda)$ at their terms in $(1 / \lambda)^{m}$, and shall define the function $\hat{w}_{q, 4}(z)$ by the formula

$$
\hat{w}_{q, 4}(z)=\hat{V}_{*}(z)-\lambda^{1-s} \hat{\Omega}(\lambda)\left[\frac{\partial}{\partial \mu} \hat{w}_{q, 3}(z, \mu)\right]_{\mu=\nu} .
$$

The relation that is obtained from (21.3) by the replacement of $L$ and $w_{q, 3}$ by $\hat{L}$ and $\hat{w}_{q, 3}$ is assured by the equation (19.6). From that, and the formula (21.6), we may derive, in the manner of (21.4), the evaluation

$$
\hat{L}\left(\hat{w}_{q, 4}, \nu, \nu\right)=\hat{L}\left(\hat{V}_{*}, \nu, \nu\right)+\lambda^{1-s} \hat{\Omega}(\lambda)\left[\frac{\partial}{\partial \mu} \hat{L}\left(\hat{w}_{q, 3}(z, \nu), \nu, \mu\right)\right]_{\mu=\nu} .
$$

The functions $\hat{V}_{*}(z), \hat{w}_{q, 3}(z, \nu)$ and $\hat{\Omega}(\lambda)$ are polynomials in $1 / \lambda$ which coincide with the formal series $V_{*}(z), w_{3}(z)$ and $\Omega(\lambda)$ to their terms in $(1 / \lambda)^{m}$. Because of that the right-hand members of the relations (21.4) and (21.7), when they are multiplied by $\lambda^{-2}$, similarly coincide. In accordance with (21.5), therefore,

$$
\lambda^{-2} \hat{L},\left(\hat{\mathfrak{u}}_{q, 4}, \nu, \nu\right)=\sum_{n=m_{0}}^{m} \frac{c_{n}}{\lambda^{n}}+\frac{E(z, \lambda)}{\lambda^{m+1}},
$$


whereas from this it may be concluded that

$$
\frac{\hat{L}^{\prime}\left(\hat{w}_{q, 4}, \nu, \nu\right)}{\hat{L}\left(\hat{w}_{q, 4}, \nu, \nu\right)}=\frac{E(z, \lambda)}{\lambda^{m-m_{0}+1}} .
$$

We may proceed now as in $\S 20$. The differential equation

$$
\hat{L}^{\prime}(w, \nu, \nu)-\frac{\hat{L}^{\prime}\left(\hat{w}_{q, 4}, \nu, \nu\right)}{\hat{L}\left(\hat{w}_{q, 4}, \nu, \nu\right)} \hat{L}(w, \nu, \nu)=0,
$$

is of the fourth order, and is solved by each of the functions $\hat{w}_{3}(z)$ and $\hat{w}_{q, j}(z)$, $j=1,2,4$. The evaluations (20.7) and (20.8), combined with (21.9), show it to have the form (20.9). It is a related equation.

\section{REFERENCES}

1. R. E. Langer, On the asymptotic solutions of a class of ordinary differential equations of the fourth order, with special reference to an equation of hydrodynamics, Trans. Amer. Math. Soc. vol. 84 (1957) pp. 144-191.

2. Wolfgang Wasow, On small disturbances of plane couette flow, J. Res. Nat. Bur. Standards, vol. 51 (1953) pp. 195-202.

3. C. C. Lin, On uniformly valid asymptotic solutions of the Orr-Sommerfeld equation, IX' Congrès International de Mécanique Appliquée, Actes, vol. 1, 1957, pp. 136-148.

4. R. E. Langer, The solutions of the differential equation $v^{\prime \prime \prime}+\lambda^{2} z v^{\prime}+3 \mu \lambda^{2} v=0$, Duke Math. J. vol. 22 (1955) pp. 525-542.

Mathematics Research Center, United States Army, MADISON, Wis. 